\title{
High accurate convergent spectral Galerkin methods for nonlinear weakly singular Volterra integro-differential equations
}

\author{
Yin Yang ${ }^{1} \cdot$ Guoting Deng $^{2} \cdot$ Emran $^{\text {Tohidi }^{3}}{ }^{\text {D }}$
}

Received: 16 October 2020 / Revised: 12 January 2021 / Accepted: 23 February 2021 /

Published online: 20 April 2021

(c) SBMAC - Sociedade Brasileira de Matemática Aplicada e Computacional 2021

\begin{abstract}
This paper contributes to investigate the Jacobi spectral and pseudo-spectral Galerkin techniques to solve a general form of nonlinear weakly singular Volterra integro-differential equations of the first order. By applying some suitable change of variables, we have made the solution of the mentioned equations to be smooth. Then, by applying the spectral and pseudo-spectral Jacobi Galerkin schemes, accurate solutions are computed efficiently. Rigorous convergence analysis associated with both the spectral and pseudo-spectral Jacobi Galerkin approaches are discussed in detail. Some numerical test problems are given to depict the accuracy of the presented numerical schemes with respect to some recent approximate methods in the literature.
\end{abstract}

Keywords Volterra integro-differential equations · Weakly singular · Spectral Galerkin technique $\cdot$ Convergence analysis

Mathematics Subject Classification 33C45 - 65B99 - 65L20 - 42C10

Communicated by Hui Liang.

Yin Yang

yangyinxtu@xtu.edu.cn

$凶 \quad$ Emran Tohidi

emrantohidi@kub.ac.ir

1 School of Mathematics and Computational Science, Xiangtan University, Hunan National Applied Mathematics Center, Xiangtan 411105, Hunan, China

2 Hunan Key Laboratory for Computation and Simulation in Science and Engineering, Key Laboratory of Intelligent Computing and Information Processing of Ministry of Education, School of Mathematics and Computational Science, Xiangtan University, Xiangtan 411105, Hunan, China

3 Department of Mathematics, Kosar University of Bojnord, P. O. Box 9415615458, Bojnord, Iran 


\section{Introduction}

The solution of differential equations and integral equations (IEs) is of great significance. Many practical problems can be analyzed and solved as mathematical models to help scientists better understand practical problems. Recently, Yel G et al. applied the sine-Gordon expansion method to shallow water wave models which are Kadomtsev-Petviashvili-Benjamin-BonaMahony and the Benney-Luke equations (Yel et al. 2020). Gao W et al. studied a deathly disease spreading pregnant women called Lassa hemorrhagic fever disease (Gao et al. 2020a) and focused on 2019-nCov systems (Gao et al. 2020b). In addition, our paper mainly consider the nonlinear weakly singular Volterra integro-differential equations (WSVIDEs). Due to the considerable importance of WSVIDEs of the first kind in modeling of physical and chemical processes in real world applications, scientists proposed some new and modern techniques to solve them accurately. As an important application of such these equations, one can point out the fractional differential equations in the Caputo or Riemann Liouville notion that can be transformed into the WSVIDEs of the first order. In this paper, we have considered the following nonlinear WSVIDEs:

$$
\begin{aligned}
& u^{\prime}(t)=g(t, u(t))+\int_{0}^{t}(t-\eta)^{-\mu} K\left(t, \eta, u(\eta), u^{\prime}(\eta)\right) d \eta, \quad t \in[0, T], \\
& u(0)=u_{0},
\end{aligned}
$$

where $0<\mu<1, g(t, u(t)) \in C\left([0, T] \times D_{R}\right), K\left(t, \eta, u(\eta), u^{\prime}(\eta)\right) \in C(D \times$ $\left.\left(D_{R}\right)^{2}\right), D:=\{(t, \eta) \mid 0 \leq \eta \leq t \leq T\}$, in which $D_{R}$ is a bounded set in real numbers. Our basic tool is based upon the Jacobi polynomials and also the spectral and pseudo-spectral Galerkin methods.

Spectral methods are the best approximate tool for WSVIDEs with smooth solutions (Shen et al. 2011). They belong to the global numerical methods and have great popularity with respect to some low order methods such as finite difference methods (FDMs). As far as we know, two basic and efficient tools for numerical techniques are operational matrices for computing the derivatives (however some operational matrices were implemented for integration) and also the high accurate Gauss quadrature rules (in different kinds of finite or infinite) for computing the definite integrals approximately. Because of the importance of IEs and integro-differential equations (IDEs), researchers have focused on the high accurate numerical solutions such as spectral methods for computing accurate solutions. A huge size of research works have been devoted to this aim, but some of them are empty from the theoretical analysis in convergence, consistency and stability of the spectral methods. Even, many research works proposed high accurate numerical methods and investigated errors estimation of the approximation for single or multi-variable functions which are independent of the proposed algorithms. Actually, the lack of proving the convergence of the proposed numerical solutions to the exact solutions theoretically is the basic disadvantage of the spectral methods for solving IEs before the year 2008. As the first primary research work that implemented the Legendre spectral collocation method (LSCM) for solving linear Volterra integral equations (VIEs), both numerically and theoretically, one can point out to Tang et al. (2008). In this research work, a high accurate numerical technique together with the associated detailed convergence analysis are investigated for solving the linear variant of VIEs. After that, a huge size of research works generalized and modified the method that implemented in Tang et al. (2008) for solving different types of problems.

As an important challenge in the numerical solution of IEs and IDEs, one may deal with weakly singularity in the solutions of VIEs. As the first attempt, Tang and Chen used LSCM 
(Chen and Tang 2009) and Jacobi spectral collocation method (JSCM) (Chen and Tang 2010) in 2009 and 2010, respectively for solving linear weakly singular Volterra integral equations (WSVIEs). After that, in 2012, the first-order linear WSVIDEs were solved by LSCM approximately (Wei and Chen 2012). One year later, in 2013, linear WSVIEs with vanishing pantograph delay was considered by JSCM rigorously (Zhang et al. 2013). In (2018), Yang X et al. solved nonlinear fractional integral differential equations based on the JSCM of Lagrange interpolation polynomials. Yang et al. (2019a) investigated the spectral collocation method for Fredholm integral equations of the second kind with a weakly singular kernel in 2019. Also (1) is approximately solved by JSCM with a rigorous convergence analysis (Shi et al. 2019). In (2021), Yang and Tang presented the mapped Laguerre spectral method for solving the Volterra integral equation with the noncompact kernel. All of these research works belong to the category of spectral collocation methods. For the spectral Galerkin method, in 2014, Zhang and Li (2014) used Jacobi pseudo-spectral Galerkin method to solve the second type of WSVIDEs. Yang Y proposed general spectral and pseudo-spectral Jacobi Galerkin methods for fractional-order integro-differential equations of Volterra type in 2015 (Yang 2015). In 2019, Yang et al. (2019b) discussed the strict convergence analysis of the Jacobi spectral Galerkin method of VIEs with noncompact kernel. It should be noted that many investigations are done in this category and Jacobi spectral Galerkin methods have had few results in this regard. This motivates us to propose Jacobi spectral and pseudo-spectral Galerkin methods for solving the equations in the form of (1).

The structure of this research article is as follows. In the Sect. 2, some appropriate change of variables are considered to make the solution of (1) to be smooth and then both of the spectral and pseudo-spectral Jacobi Galerkin techniques are applied to solve the regularized form of the equation (1). Some useful lemmas are introduced (from the literature), that have a crucial rule in proving the main theorems in Sect. 3. In Sect. 4, rigorous convergence analysis associated with the proposed schemes are investigated in details. An extensive set of test problems are provided in Sect. 5 and also some comparison are made with recent numerical methods in the literature. From the Figures and Tables in Sect. 5 we can conclude efficiency and accuracy of the suggested numerical schemes with respect to other numerical approaches. Some conclusions and plans for our future research works are given in Sect. 6.

\section{Spectral Galerkin method}

In this section, we need to use the variable transformation to deal with the equations (1). Because we consider the equations with the non-smooth solution. Regularity of Volterratype integral equation or integral differential equation solution and application of collocation method lies at the heart of the discussion on Brunner (2004), in which the behavior of the $n$-th derivative of the equations (1) solution $u(t)$ is similar to $u^{(n)}(t) \sim t^{2-\mu-n}$ when $t \rightarrow 0$. It also show that $u \notin C^{n+1}(\Lambda)$. Thus, we are inspired by Baratella and Orsi (2004), Sohrabi et al. (2017), Lü and Huang (2006), the authors changed the original equation into another equation which is also weakly singular by applying a variable transformation, but the solution is smooth. Thus, set $t=\delta(s)=s^{p}, \eta=\delta(\xi)=\xi^{p}$, we get

$$
\begin{aligned}
& \hat{u}^{\prime}(s)=\hat{g}(s, \hat{u}(s))+\int_{0}^{s}(\delta(s)-\delta(\xi))^{-\mu} \hat{K}\left(s, \xi, \hat{u}(\xi), \hat{u}^{\prime}(\xi)\right) d \xi, \quad s \in[0, \sqrt[p]{T}], \\
& \hat{u}(0)=u_{0} .
\end{aligned}
$$

where $p$ is a positive integer and 


$$
\begin{aligned}
& \hat{u}(s)=u(\delta(s)), \quad \hat{g}(s, \hat{u}(s))=\delta^{\prime}(s) g(\delta(s), u(\delta(s))), \\
& \hat{K}\left(s, \xi, \hat{u}(\xi), \hat{u}^{\prime}(\xi)\right)=\delta^{\prime}(s) \delta^{\prime}(\xi) K\left(\delta(s), \delta(\xi), \hat{u}(\xi), \frac{1}{\delta^{\prime}(\xi)} \hat{u}^{\prime}(\xi)\right) .
\end{aligned}
$$

Rewrite the equation (2) as

$$
\begin{aligned}
& \hat{u}^{\prime}(s)=\hat{g}(s, \hat{u}(s))+\int_{0}^{s}(s-\xi)^{-\mu} \bar{K}\left(s, \xi, \hat{u}(\xi), \hat{u}^{\prime}(\xi)\right) d \xi, \quad s \in[0, \sqrt[p]{T}], \\
& \hat{u}(0)=u_{0}
\end{aligned}
$$

where

$$
\bar{K}\left(s, \xi, \hat{u}(\xi), \hat{u}^{\prime}(\xi)\right)=\left(\frac{\delta(s)-\delta(\xi)}{s-\xi}\right)^{-\mu} \hat{K}\left(s, \xi, \hat{u}(\xi), \hat{u}^{\prime}(\xi)\right), \quad s \neq \xi
$$

and the $n$-th derivative of the equation (3) solution satisfies $\hat{u}^{(n)}(s) \sim s^{p(2-\mu)-n}, s \rightarrow 0$. It means that we can choose a suitable $p$ such that $\hat{u}(s)$ is smooth. And once $\hat{u}(s), \hat{u}^{\prime}(s)$ of the transformed equation (3) is known, $u(t), u^{\prime}(t)$ of the original equation (1) can be obtained by inverse transformation, namely

$$
u(t)=\hat{u}\left(\delta^{-1}(t)\right), u^{\prime}(t)=\frac{\hat{u}^{\prime}\left(\delta^{-1}(t)\right)}{\delta^{\prime}\left(\delta^{-1}(t)\right)},
$$

where $\delta^{-1}(t)$ is the inverse transformation corresponding to $\delta(s)$. It should be noted that $\delta(s)$ is reversible within the selected interval.

Next, in order to solve more conveniently and apply the theory of orthogonal polynomials, we use the following linear transformation

$$
s=\frac{\sqrt[p]{T}}{2}(1+z), \quad \xi=\frac{\sqrt[p]{T}}{2}(1+\sigma),
$$

and rewrite (3) as follows

$$
\begin{aligned}
& y^{\prime}(z)=f(z, y(z))+\int_{-1}^{z}(z-\sigma)^{-\mu} \widetilde{K}\left(z, \sigma, y(\sigma), y^{\prime}(\sigma)\right) d \sigma, \quad z \in \Lambda:=[-1,1], \\
& y(-1)=y_{-1}=u_{0} .
\end{aligned}
$$

where

$$
\begin{aligned}
& y(z)=\hat{u}\left(\frac{\sqrt[p]{T}}{2}(1+z)\right), \quad f(z, y(z))=\frac{\sqrt[p]{T}}{2} \hat{g}\left(\frac{\sqrt[p]{T}}{2}(1+z), y(z)\right), \\
& \widetilde{K}\left(z, \sigma, y(\sigma), y^{\prime}(\sigma)\right)=\left(\frac{\sqrt[p]{T}}{2}\right)^{2-\mu} \bar{K}\left(\frac{\sqrt[p]{T}}{2}(1+z), \frac{p}{2}(1+\sigma), y(\sigma), \frac{2}{\sqrt[p]{T}} y^{\prime}(\sigma)\right) .
\end{aligned}
$$

For the convenience of numerical implementation and analysis, the initial condition is reformulated as

$$
y(z)=y_{-1}+\int_{-1}^{z} y^{\prime}(\sigma) d \sigma
$$


Furthermore, in order to simplify notation and facilitate the subsequent convergence analysis, we introduce the integral operator and some symbols as follows

$$
\begin{aligned}
& \left(\mathcal{K}\left(y, y^{\prime}\right)\right)(z)=\int_{-1}^{z}(z-\sigma)^{-\mu} \widetilde{K}\left(z, \sigma, y(\sigma), y^{\prime}(\sigma)\right) d \sigma, \quad \mathcal{K}: C(\Lambda) \rightarrow C(\Lambda), \\
& (\mathcal{H} y)(z)=\int_{-1}^{z} y(\sigma) d \sigma, \quad \mathcal{H}: C(\Lambda) \rightarrow C(\Lambda), \\
& (\mathcal{F} y)(z)=f(z, y(z)) .
\end{aligned}
$$

Then, (4) and (5) can be reformulated as

$$
\begin{aligned}
& y^{\prime}(z)=(\mathcal{F} y)(z)+\left(\mathcal{K}\left(y, y^{\prime}\right)\right)(z), \\
& y(z)=y_{-1}+\left(\mathcal{H} y^{\prime}\right)(z), \quad z \in \Lambda .
\end{aligned}
$$

\subsection{Jacobi spectral Galerkin method}

For this subsection, we will investigate the numerical fulfillment of the Jacobi spectral Galerkin technique to solve equations (6). First, we will give some symbol definitions. For $\forall N \in \mathbb{N}, P_{N}$ indicates the space consisting of polynomials defined on $\Lambda$ with degree not exceeding $N$ and $\mathbb{N}$ represents the set of all non-negative integers. In addition, we suppose that $J_{k}(x)$ is the $k$-th Jacobi polynomial related to the weight function $\omega^{\theta, v}(z)=$ $(1-z)^{\theta}(1+z)^{v}(\theta, v>-1)$. Then, we have $\mathcal{P}_{N}=\operatorname{span}\left\{J_{0}(z), J_{1}(z), \ldots, J_{N}(z)\right\}$.

Next, implementing the Jacobi spectral Galerkin approach to solve (6) can be expressed as: find $y_{N}, y_{N}^{\prime} \in \mathcal{P}_{N}$, such that

$$
\left\{\begin{array}{l}
\left(y_{N}^{\prime}, \psi_{N}\right)_{\omega^{\theta, v}}=\left(\mathcal{F} y_{N}, \psi_{N}\right)_{\omega^{\theta, v}}+\left(\mathcal{K}\left(y_{N}, y_{N}^{\prime}\right), \psi_{N}\right)_{\omega^{\theta, v}} \\
\left(y_{N}, \psi_{N}\right)_{\omega^{\theta, v}}=\left(y_{-1}, \psi_{N}\right)_{\omega^{\theta, v}}+\left(\mathcal{H} y_{N}^{\prime}, \psi_{N}\right)_{\omega^{\theta, v}}
\end{array}, \forall \psi_{N} \in \mathcal{P}_{N} .\right.
$$

where $(y, \psi)_{\omega^{\theta, v}}=\int_{-1}^{1} y(z) \psi(z) \omega^{\theta, v}(z) d z$ is the continuous inner product.

And then, we set $y(z) \approx y_{N}(z)=\sum_{k=0}^{N} y_{k} J_{k}(z), y^{\prime}(z) \approx y_{N}^{\prime}(z)=\sum_{k=0}^{N} y_{k}^{\prime} J_{k}(z)$ and take $\psi_{N}(z)=J_{l}(z), z \in[-1,1]$, yields

$$
\begin{aligned}
& \sum_{k=0}^{N} y_{k}^{\prime}\left(J_{k}, J_{l}\right)_{\omega^{\theta, v}}=\left(\mathcal{F}\left(\sum_{k=0}^{N} y_{k} J_{k}\right), J_{l}\right)_{\omega^{\theta, v}}+\left(\mathcal{K}\left(\sum_{k=0}^{N} y_{k} J_{k}, \sum_{k=0}^{N} y_{k}^{\prime} J_{k}\right), J_{l}\right)_{\omega^{\theta, v}}, \\
& \sum_{k=0}^{N} y_{k}\left(J_{k}, J_{l}\right)_{\omega^{\theta, v}}=\left(y_{-1}, J_{l}\right)_{\omega^{\theta, v}}+\sum_{k=0}^{N} y_{k}^{\prime}\left(\mathcal{H} J_{k}, J_{l}\right)_{\omega^{\theta, v}}, \quad l=0,1, \ldots, N .
\end{aligned}
$$

Actually, (8) is a system of nonlinear algebraic equations (SNAEs). It can be solved by iterative solvers such as Newton-Raphson algorithm in different softwares.

\subsection{Jacobi pseudo-spectral Galerkin method}

For this subsection, we will demonstrate the numerical implementation of the Jacobi pseudospectral Galerkin technique to solve (6). Set

$$
\sigma(z, \zeta)=\frac{1+z}{2} \zeta+\frac{z-1}{2}, \quad-1 \leq \zeta \leq 1
$$


Then, we obtain

$$
\begin{aligned}
\left(\mathcal{K}\left(y, y^{\prime}\right)\right)(z) & =\int_{-1}^{z}(z-\sigma)^{-\mu} \widetilde{K}\left(z, \sigma, y(\sigma), y^{\prime}(\sigma)\right) d \sigma \\
& =\left(\frac{1+z}{2}\right)^{1-\mu} \int_{-1}^{1}(1-\zeta)^{-\mu} \widetilde{K}\left(z, \sigma(z, \zeta), y(\sigma(z, \zeta)), y^{\prime}(\sigma(z, \zeta))\right) d \zeta, \\
\left(\mathcal{H} y^{\prime}\right)(z) & =\int_{-1}^{z} y^{\prime}(\sigma) d \sigma=\frac{1+z}{2} \int_{-1}^{1} y^{\prime}(\sigma(z, \zeta)) d \zeta .
\end{aligned}
$$

Applying $(N+1)$-points Gauss quadrature rules to approximate the integral terms involved in (9) yields

$$
\begin{aligned}
& \left(\mathcal{K}\left(y, y^{\prime}\right)\right)(z) \approx\left(\mathcal{K}_{N}\left(y, y^{\prime}\right)\right)(z)=\left(\frac{1+z}{2}\right)^{1-\mu} \sum_{i=0}^{N} \widetilde{K}\left(z, \sigma\left(z, \zeta_{i}\right),\right. \\
& \left.y\left(\sigma\left(z, \zeta_{i}\right)\right), y^{\prime}\left(\sigma\left(z, \zeta_{i}\right)\right)\right) \omega_{i}^{-\mu, 0} \\
& \left(\mathcal{H} y^{\prime}\right)(z) \approx\left(\mathcal{H}_{N} y^{\prime}\right)(z)=\frac{1+z}{2} \sum_{i=0}^{N} y^{\prime}\left(\sigma\left(z, \varsigma_{i}\right)\right) \omega_{i}^{0,0} .
\end{aligned}
$$

where $\left\{\zeta_{i}\right\}_{i=0}^{N},\left\{\zeta_{i}\right\}_{i=0}^{N}$ are the $(N+1)$-degree Jacobi-Gauss points, and $\left\{\omega_{i}^{-\mu, 0}\right\}_{i=0}^{N}$, $\left\{\omega_{i}^{0,0}\right\}_{i=0}^{N}$ are the corresponding Jacobi-Gauss weights, respectively.

Furthermore, we apply the discrete inner product to replace continuous inner products in (7), (8), namely

$$
(y, v)_{\omega^{\theta, v}} \approx(y, v)_{\omega^{\theta, \nu}, N}=\sum_{j=0}^{N} y\left(z_{j}\right) v\left(z_{j}\right) \omega_{j}^{\theta, v}, \quad 0 \leq j \leq N .
$$

where $\left\{z_{j}\right\}_{j=0}^{N}$ and $\left\{\omega_{j}^{\theta, v}\right\}_{j=0}^{N}$ are the $(N+1)$-degree Jacobi-Gauss points and their relevant Jacobi weights, respectively. In addition, we get

$$
(y, v)_{\omega^{\theta, v}}=(y, v)_{\omega^{\theta, v}, N}, \quad \text { if } y v \in \mathcal{P}_{2 N+1} .
$$

Thus, we substitute (10) and (11) into (7). And, the Jacobi pseudo-spectral Galerkin technique is find $y(z) \approx \bar{y}_{N}(z)=\sum_{k=0}^{N} \bar{y}_{k} J_{k}(z), y^{\prime}(z) \approx \bar{y}_{N}^{\prime}(z)=\sum_{k=0}^{N} \bar{y}_{k}^{\prime} J_{k}(z)$, such that

$$
\left\{\begin{array}{l}
\left(\bar{y}_{N}^{\prime}, \psi_{N}\right)_{\omega^{\theta, v}, N}=\left(\mathcal{F} \bar{y}_{N}, \psi_{N}\right)_{\omega^{\theta, v}, N}+\left(\mathcal{K}_{N}\left(\bar{y}_{N}, \bar{y}_{N}^{\prime}\right), \psi_{N}\right)_{\omega^{\theta, v}, N}, \quad \forall \psi_{N} \in \mathcal{P}_{N} . \\
\left(\bar{y}_{N}, \psi_{N}\right)_{\omega^{\theta, v}, N}=\left(y_{-1}, \psi_{N}\right)_{\omega^{\theta, v}, N}+\left(\mathcal{H}_{N} \bar{y}_{N}^{\prime}, \psi_{N}\right)_{\omega^{\theta, \nu}, N} .
\end{array}\right.
$$

we also take $\psi_{N}(z)=J_{l}(z), z \in[-1,1]$, yields

$$
\begin{aligned}
\sum_{k=0}^{N} \bar{y}_{k}^{\prime}\left(J_{k}, J_{l}\right)_{\omega^{\theta, \nu}, N}= & \left(\mathcal{F}\left(\sum_{k=0}^{N} \bar{y}_{k} J_{k}\right), J_{l}\right)_{\omega^{\theta, \nu}, N} \\
& +\left(\mathcal{K}_{N}\left(\sum_{k=0}^{N} \bar{y}_{k} J_{k}, \sum_{k=0}^{N} \bar{y}_{k}^{\prime} J_{k}\right), J_{l}\right)_{\omega^{\theta, \nu}, N}, \\
\sum_{k=0}^{N} \bar{y}_{k}\left(J_{k}, J_{l}\right)_{\omega^{\theta, \nu}, N}= & \left(y_{-1}, J_{l}\right)_{\omega^{\theta, \nu}, N}+\sum_{k=0}^{N} \bar{y}_{k}^{\prime}\left(\mathcal{H}_{N} J_{k}, J_{l}\right)_{\omega^{\theta, \nu}, N}, \quad l=0,1, \ldots, N .
\end{aligned}
$$


Similarly, we obtain a SNAEs (13). It can be solved by iterative solvers in different softwares.

\section{Preliminaries}

For this section, we will introduce certain related lemmas it is significant for the construction of the theorems in the next section. In addition, throughout this paper, $C$ is a positive constant independent of $N$ and it is clear to know the specific meaning according to the context.

First of all, we define the operator of Jacobi orthogonal projection $\Pi_{N}^{\theta, v}: L_{\omega^{\theta, v}}^{2}(\Lambda) \rightarrow \mathcal{P}_{N}$, which satifies

$$
\left(\Pi_{N}^{\theta, v} u, v_{N}\right)_{\omega^{\theta, v}}=\left(u, v_{N}\right)_{\omega^{\theta, v}}, \quad \forall u \in L_{\omega^{\theta, v}}^{2}(\Lambda), v_{N} \in \mathcal{P}_{N} .
$$

Next, set $L_{\omega^{\theta, v}}^{2}(\Lambda)$ is the measurable functions space and its norm as follows

$$
\|u\|_{\omega^{\theta, v}}:=\left(\int_{\Lambda} u^{2}(z) \omega^{\theta, v}(z) d z\right)^{\frac{1}{2}} .
$$

$L^{\infty}(\Lambda)$ represents the measurable functions space and the norm is defined in the following form

$$
\|u\|_{\infty}:=\underset{z \in \Lambda}{\operatorname{ess} \sup }|u(z)|
$$

Further, define

$$
H_{\omega^{\theta, v}}^{m}(\Lambda)=\left\{u: D^{j} u:=\frac{d^{j} u}{d z^{j}} \in L_{\omega^{\theta, v}}^{2}(\Lambda), 0 \leq j \leq m\right\},
$$

equipped with the norm and semi-norm

$$
\begin{aligned}
\|u\|_{H^{\theta, v}}^{m}(\Lambda) & =\left(\sum_{j=0}^{m}\left\|D^{j} u\right\|_{\omega^{\theta, v}}^{2}\right)^{\frac{1}{2}}, \\
|u|_{H_{\omega^{\theta, v}}^{m ; N}(\Lambda)} & =\left(\sum_{j=\min (m, N+1)}^{m}\left\|D^{j} u\right\|_{\omega^{\theta, v}}^{2}\right)^{\frac{1}{2}} .
\end{aligned}
$$

When $\omega^{\theta, v}(z)=1$, then $L_{\omega^{\theta, v}}^{2}(\Lambda), H_{\omega^{\theta, v}}^{m}(\Lambda)$ and $\|\cdot\|_{\omega^{\theta, v}}$ are denoted simply by $L^{2}(\Lambda), H^{m}(\Lambda),\|\cdot\|$, respectively.

Lemma 3.1 (Tao et al. 2011) Assume that $u \in H_{\omega^{\theta, v}}^{m}(\Lambda)$ and $m \geq 1$.

(i) If $\theta, v>-1$, then

$$
\left\|u-\Pi_{N}^{\theta, v} u\right\|_{\omega^{\theta, v}} \leq C N^{-m}|u|_{H_{\omega^{\theta, v}}^{m ; N}},
$$

(ii) If $-1<\theta, v \leq 0$, then

$$
\left\|u-\Pi_{N}^{\theta, v} u\right\|_{\infty} \leq C N^{\frac{3}{4}-m}|u|_{H_{\omega}^{\theta, v}} .
$$


Lemma 3.2 (Shen et al. 2011; Canuto et al. 2006) Presume that $u \in H_{\omega^{\theta, v}}^{m}(\Lambda), m \geq 1, I_{N}^{\theta, v} u$ represent the interpolation operator of $u$ depended on $(N+1)$ degree Jacobi Gauss points which related to weight function $\omega^{\theta, v}(z)$ with $\theta, v>-1$, namely,

$$
I_{N}^{\theta, v} u(z)=\sum_{i=0}^{N} u\left(z_{i}\right) F_{i}(z),
$$

where $F_{i}$ denotes the ith Lagrange basis function. Then

$$
\begin{aligned}
& \left\|u-I_{N}^{\theta, v} u\right\|_{\omega^{\theta, v}} \leq C N^{-m}|u|_{H_{\omega^{\theta, v}}^{m ; N}}, \\
& \left\|u-I_{N}^{\theta, v} u\right\|_{\infty} \leq\left\{\begin{array}{l}
C N^{\frac{1}{2}-m} \log N|u|_{H_{\omega^{c}}^{m ; N}}, \quad-1<\theta, v \leq-\frac{1}{2}, \\
C N^{\gamma+1-m}|u|_{H^{m}{ }^{m} ; N}, \quad \text { otherwise, } \gamma=\max \{\theta, v\},
\end{array}\right.
\end{aligned}
$$

where $\omega^{c}=\omega^{-\frac{1}{2},-\frac{1}{2}}$ is the Chebyshev weight function.

Lemma 3.3 (Mastroianni and Occorsio 2001) Suppose that $u$ is a bounded function, then there exists a constant $C$ not depend on $N$ such that

$$
\sup _{N}\left\|I_{N}^{\theta, v} u\right\|_{\omega^{\theta, v}} \leq C\|u\|_{\infty} .
$$

Lemma 3.4 (Mastroianni and Occorsio 2001) Suppose that $\left\{F_{j}(z)\right\}_{j=0}^{N}$ represent the Lagrange basis polynomials relevant to Jacobi Gauss points. Then

$$
\left\|I_{N}^{\theta, v}\right\|_{\infty}=\max _{z \in[-1,1]} \sum_{j=0}^{N}\left|F_{j}(z)\right|= \begin{cases}O(\log N), & -1<\theta, v \leq-\frac{1}{2}, \\ O\left(N^{\gamma+\frac{1}{2}}\right), & \gamma=\max \{\theta, v\}, \text { otherwise. }\end{cases}
$$

Lemma 3.5 (Shen et al. 2011; Canuto et al. 2006) Assume that it apply the $(N+1)$-points Jacobi Gauss quadrature rule to approximate the product $u \psi$, where $u \in H_{\omega^{\theta, v}}^{m}(\Lambda)$ with $m \geq 1$ and $\psi \in P_{N}$. Then

$$
\left|(u, \psi)_{\omega^{\theta, \nu}}-(u, \psi)_{\omega^{\theta, \nu}, N}\right| \leq C N^{-m}|u|_{H_{\omega^{\theta, \nu}}^{m ; N}}\|\psi\|_{\omega^{\theta, \nu}},
$$

Lemma 3.6 (Willett 1965) Suppose that $0 \leq M_{2}, M_{3} \leq+\infty, \mu \in(0,1)$, and $u(z)$ is a nonnegative locally integrable function, satisfying

$$
u(z) \leq M_{2} \int_{-1}^{z} u(\sigma) d \sigma+M_{3} \int_{-1}^{z}(z-\sigma)^{-\mu} u(\sigma) d \sigma+G(z), \quad z \in[-1,1],
$$

where $G(z)$ is also an locally integrable function, then it exists a constant $C$ such that

$$
u(z) \leq C \int_{-1}^{z} G(\sigma) d \sigma+C \int_{-1}^{z}(z-\sigma)^{-\mu} G(\sigma) d \sigma+G(z), \quad z \in[-1,1] .
$$

Next, we will introduce the $H \ddot{o l}$ der space. Set $m \geq 0$ and $\varrho \in(0,1), C^{m, \varrho}(\Lambda)$ consists of the $m$-times continuously differentiable function $u$ and whose $m$-th derivatives are Hölder continuous with exponent $\varrho$. The norm is defined as follows:

$$
\|u\|_{m, \varrho}=\sum_{k=0}^{m} \max _{z \in \Lambda}\left|\partial_{z}^{k} u(z)\right|+\sup _{\substack{z_{1}, z_{2} \in \Lambda \\ z_{1} \neq z_{2}}} \frac{\left|\partial_{z}^{m} u\left(z_{1}\right)-\partial_{z}^{m} u\left(z_{2}\right)\right|}{\left|z_{1}-z_{2}\right|^{\varrho}} .
$$


and if $\varrho=0$, then $C^{m, 0}(\Lambda)$ represents the space of the $m$-times continuously derivative functions on $\Lambda$, it is also generally indicated by $C^{m}(\Lambda)$, and with norm $\|\cdot\|_{m}$.

We will apply the results of Ragozin (1970, 1971), namely, for $m \geq 0$ and $0<\varrho<1$, there exists a constant $\mathcal{C}_{m, \varrho}>0$ such that $\forall u \in C^{m, \varrho}(\Lambda)$, then there exists a polynomial function $\mathfrak{L}_{N} u \in \mathcal{P}_{N}$ such that

$$
\left\|u-\mathfrak{L}_{N} u\right\|_{\infty} \leq \mathcal{C}_{m, \varrho} N^{-(m+\varrho)}\|u\|_{m, \varrho} .
$$

where $\mathfrak{L}_{N}$ is a linear operator from $C^{m, \varrho}(\Lambda)$ into $\mathcal{P}_{N}$.

Lemma 3.7 Let $\varrho \in(0,1), H(z, \sigma), \frac{\partial H(z, \sigma)}{\partial z} \in C(\Lambda)$ and the linear operator $\mathcal{M}$ be defined by

$$
(\mathcal{M} u)(z)=\int_{-1}^{z}(z-\sigma)^{-\mu} H(z, \sigma) u(\sigma) d \sigma,
$$

Then, $\forall u \in C(\Lambda)$, there exists a non-negative constant $C$ such that

$$
\|\mathcal{M} u\|_{0, \varrho} \leq C\|u\|_{\infty} .
$$

which implies that $\mathcal{M}$ is compact as operator from $C(\Lambda)$ to $C^{0, \varrho}(\Lambda)$.

Proof First, assume that $0<\varrho<1-\mu$, for any $z_{1}, z_{2} \in \Lambda$ and $z_{1} \neq z_{2}$. Due to the definition, we have

$$
\|\mathcal{M} u\|_{0, \varrho}=\max _{z \in \Lambda}|\mathcal{M} u(z)|+\sup _{\substack{z_{1}, z_{2} \in \Lambda \\ z_{1} \neq z_{2}}} \frac{\left|\mathcal{M} u\left(z_{1}\right)-\mathcal{M} u\left(z_{2}\right)\right|}{\left|z_{1}-z_{2}\right|^{\varrho}},
$$

For the first term of the above equation, we have

$$
\begin{aligned}
|\mathcal{M} u(z)| & =\left|\int_{-1}^{z}(z-\sigma)^{-\mu} H(z, \sigma) u(\sigma) d \sigma\right| \leq\|H\|_{\infty} \int_{-1}^{z}(z-\sigma)^{-\mu}|u(\sigma)| d \sigma \\
& \leq C\|u\|_{\infty} \int_{-1}^{z}(z-\sigma)^{-\mu} d \sigma \leq C \frac{2^{1-\mu}}{1-\mu}\|u\|_{\infty} \leq C\|u\|_{\infty}
\end{aligned}
$$

Thus, we get $\max _{z \in \Lambda}|\mathcal{M} u(z)| \leq C\|u\|_{\infty}$.

Without loss of generality, we presume that $-1 \leq z_{1}<z_{2} \leq 1$. Then

$$
\begin{aligned}
& \frac{\left|\mathcal{M} u\left(z_{1}\right)-\mathcal{M} u\left(z_{2}\right)\right|}{\left|z_{1}-z_{2}\right|^{\varrho}}=\left(z_{2}-z_{1}\right)^{-\varrho} \mid \int_{-1}^{z_{1}}\left(z_{1}-\eta\right)^{-\mu} H\left(z_{1}, \eta\right) u(\eta) d \eta \\
& \quad-\int_{-1}^{z_{2}}\left(z_{2}-\eta\right)^{-\mu} H\left(z_{2}, \eta\right) u(\eta) d \eta \mid \\
& \leq\left(z_{2}-z_{1}\right)^{-\varrho}\left|\int_{-1}^{z_{1}}\left(z_{1}-\eta\right)^{-\mu}\left(H\left(z_{1}, \eta\right)-H\left(z_{2}, \eta\right)\right) u(\eta) d \eta\right| \\
& \quad+\left(z_{2}-z_{1}\right)^{-\varrho}\left|\int_{-1}^{z_{1}}\left(\left(z_{1}-\eta\right)^{-\mu}-\left(z_{2}-\eta\right)^{-\mu}\right) H\left(z_{2}, \eta\right) u(\eta) d \eta\right| \\
& \quad+\left(z_{2}-z_{1}\right)^{-\varrho}\left|\int_{z_{1}}^{z_{2}}\left(z_{2}-\eta\right)^{-\mu} H\left(z_{2}, \eta\right) u(\eta) d \eta\right|=: Q_{1}+Q_{2}+Q_{3} .
\end{aligned}
$$

We apply the Lagrange mean value differential theorem to $Q_{1}$, we obtain

$$
Q_{1} \leq\left(z_{2}-z_{1}\right)^{-\varrho} \int_{-1}^{z_{1}}\left(z_{1}-\eta\right)^{-\mu}\left|H\left(z_{1}, \eta\right)-H\left(z_{2}, \eta\right)\right||u(\eta)| d \eta
$$




$$
\begin{aligned}
& \leq\left(z_{2}-z_{1}\right)^{1-\varrho}\left\|\frac{\partial H}{\partial z}\right\|_{\infty} \int_{-1}^{z_{1}}\left(z_{1}-\eta\right)^{-\mu}|u(\eta)| d \eta \\
& \leq C\left(z_{2}-z_{1}\right)^{1-\varrho} \frac{\left(z_{1}+1\right)^{1-\mu}}{1-\mu}\|u\|_{\infty} \leq C\left(z_{2}-z_{1}\right)^{1-\varrho}\|u\|_{\infty} .
\end{aligned}
$$

Furthermore, we estimate $Q_{2}$. Clearly

$$
\begin{aligned}
Q_{2} & \leq\left(z_{2}-z_{1}\right)^{-\varrho}\|H\|_{\infty} \int_{-1}^{z_{1}}\left|\left(z_{1}-\eta\right)^{-\mu}-\left(z_{2}-\eta\right)^{-\mu}\right||u(\eta)| d \eta \\
& \leq C\left(z_{2}-z_{1}\right)^{-\varrho}\|u\|_{\infty}\left(\frac{\left(z_{1}+1\right)^{1-\mu}}{1-\mu}+\frac{\left(z_{2}-z_{1}\right)^{1-\mu}}{1-\mu}-\frac{\left(z_{2}+1\right)^{1-\mu}}{1-\mu}\right) \\
& \leq C\left(z_{2}-z_{1}\right)^{1-\mu-\varrho}\|u\|_{\infty} .
\end{aligned}
$$

At last, we have

$$
\begin{aligned}
Q_{3} & \leq\left(z_{2}-z_{1}\right)^{-\varrho}\|H\|_{\infty} \int_{z_{1}}^{z_{2}}\left(z_{2}-\eta\right)^{-\mu}|u(\eta)| d \eta \\
& \leq C\left(z_{2}-z_{1}\right)^{-\varrho}\|u\|_{\infty} \frac{\left(z_{2}-z_{1}\right)^{1-\mu}}{1-\mu} \\
& \leq C\left(z_{2}-z_{1}\right)^{1-\mu-\varrho}\|u\|_{\infty} .
\end{aligned}
$$

Combining the above estimation formula, we get

$$
\frac{\left|\mathcal{M} u\left(z_{1}\right)-\mathcal{M} u\left(z_{2}\right)\right|}{\left|z_{1}-z_{2}\right|^{\varrho}} \leq C\left(\left(z_{2}-z_{1}\right)^{1-\varrho}+2\left(z_{2}-z_{1}\right)^{1-\mu-\varrho}\right)\|u\|_{\infty} \leq C\|u\|_{\infty},
$$

for $0<\varrho<1-\mu$.

Therefore, we prove that $\mathcal{M}$ satisfies (24).

Lemma 3.8 (Kunfer and Persson 2003) For any measurable function $v \geq 0$, the generalized Hardy's inequality as follows

$$
\left(\int_{d}^{e}|(\mathcal{G} v)(z)|^{q} \omega_{1}(z) d z\right)^{\frac{1}{q}} \leq C\left(\int_{d}^{e}|v(z)|^{p} \omega_{2}(z) d z\right)^{\frac{1}{p}}, \quad-\infty \leq d<e \leq \infty,
$$

holds if and only if

$$
\sup _{d<z<e}\left(\int_{z}^{e} \omega_{1}(t) d t\right)^{\frac{1}{q}}\left(\int_{d}^{z} \omega_{2}^{1-p^{\prime}}(t) d t\right)^{\frac{1}{p^{\prime}}}<\infty, \quad p^{\prime}=\frac{p}{p-1},
$$

where

$$
(\mathcal{G} v)(z)=\int_{d}^{z} k(z, t) v(t) d t,
$$

with $k(z, t)$ is a kernel function, $\omega_{1}, \omega_{2}$ are weight functions and $1<p \leq q<\infty$.

Lemma 3.9 (Douglas et al. 1974) Suppose that $u \in L_{\omega^{\theta, v}}^{2}(\Lambda)$, then we have

$$
\left\|\Pi_{N}^{\theta, v} u\right\|_{\omega^{\theta, v}} \leq C\|u\|_{\omega^{\theta, v}}, \quad\left\|\Pi_{N}^{\theta, v} u\right\|_{\infty} \leq C\|u\|_{\infty} .
$$




\section{Convergence analysis}

This section includes two principal parts. The first one contains the strict convergence analysis of Jacobi spectral Galerkin technique for solving (6). However, the other part investigates the convergence analysis of Jacobi pseudo-spectral Galerkin technique to solve (6).

\subsection{Jacobi spectral Galerkin method}

According to the equation (7) and the definition of the projection operator $\Pi_{N}^{\theta, v}$, the Jacobi spectral Galerkin approximate solution $y_{N}, y_{N}^{\prime}$ satisfies

$$
\left\{\begin{array}{l}
y_{N}^{\prime}=\Pi_{N}^{\theta, v} \mathcal{F} y_{N}+\Pi_{N}^{\theta, v} \mathcal{K}\left(y_{N}, y_{N}^{\prime}\right), \\
y_{N}=\Pi_{N}^{\theta, v} y_{-1}+\Pi_{N}^{\theta, v} \mathcal{H} y_{N}^{\prime} .
\end{array}\right.
$$

Theorem 4.1 Suppose that $y_{N}(z), y_{N}^{\prime}(z)$ is the Jacobi spectral approximate solution and approximate derivative of (6), which are decided by (7). If the exact solution $y(z) \in$ $H_{\omega^{\theta, v}}^{m+1 ; N}(\Lambda)(m \geq 1)$, then the error estimates hold as follows

$$
\begin{aligned}
& \left\|y^{(i)}-y_{N}^{(i)}\right\|_{\infty} \leq C N^{\frac{3}{4}-m} \mathcal{U} . \\
& \left\|y^{(i)}-y_{N}^{(i)}\right\|_{\omega^{\theta, v}} \leq C N^{-m}\left(1+N^{\frac{3}{4}-\varrho}\right) \mathcal{U} .
\end{aligned}
$$

where $\mathcal{U}=|y|_{\substack{H_{\omega}^{\theta, v} \\ m ; N}}+\left|y^{\prime}\right|_{H_{\omega}^{\theta, v}}^{m ; N}, 0<\varrho<1-\mu,-1<\theta, v \leq 0$ and $i=0,1$.

Proof Set $e=y-y_{N}, \quad e^{\prime}=y^{\prime}-y_{N}^{\prime}$.

Subtraction (25) from (6), we get

$$
\left\{\begin{array}{l}
y^{\prime}-y_{N}^{\prime}=\mathcal{F} y-\Pi_{N}^{\theta, v} \mathcal{F} y_{N}+\mathcal{K}\left(y, y^{\prime}\right)-\Pi_{N}^{\theta, v} \mathcal{K}\left(y_{N}, y_{N}^{\prime}\right), \\
y-y_{N}=\mathcal{H} y^{\prime}-\Pi_{N}^{\theta, v} \mathcal{H} y_{N}^{\prime} .
\end{array}\right.
$$

Direct computations show that

$$
\begin{aligned}
\mathcal{K} & \left(y, y^{\prime}\right)-\Pi_{N}^{\theta, v} \mathcal{K}\left(y_{N}, y_{N}^{\prime}\right) \\
= & \mathcal{K}\left(y, y^{\prime}\right)-\Pi_{N}^{\theta, v} \mathcal{K}\left(y, y^{\prime}\right)+\Pi_{N}^{\theta, v} \mathcal{K}\left(y, y^{\prime}\right)-\Pi_{N}^{\theta, v} \mathcal{K}\left(y_{N}, y_{N}^{\prime}\right) \\
= & y^{\prime}-\mathcal{F} y-\Pi_{N}^{\theta, v}\left(y^{\prime}-\mathcal{F} y\right)+\left(\mathcal{K}\left(y, y^{\prime}\right)-\mathcal{K}\left(y_{N}, y_{N}^{\prime}\right)\right) \\
& +\Pi_{N}^{\theta, v} \mathcal{K}\left(y, y^{\prime}\right)-\Pi_{N}^{\theta, v} \mathcal{K}\left(y_{N}, y_{N}^{\prime}\right)-\left(\mathcal{K}\left(y, y^{\prime}\right)-\mathcal{K}\left(y_{N}, y_{N}^{\prime}\right)\right) . \\
\mathcal{H} y^{\prime} & -\Pi_{N}^{\theta, v} \mathcal{H} y_{N}^{\prime} \\
= & \mathcal{H} y^{\prime}-\Pi_{N}^{\theta, v} \mathcal{H} y^{\prime}+\Pi_{N}^{\theta, v} \mathcal{H} y^{\prime}-\Pi_{N}^{\theta, v} \mathcal{H} y_{N}^{\prime} \\
= & y-\Pi_{N}^{\theta, v} y+\mathcal{H} y^{\prime}-\mathcal{H} y_{N}^{\prime}+\Pi_{N}^{\theta, v}\left(\mathcal{H} y^{\prime}-\mathcal{H} y_{N}^{\prime}\right)-\left(\mathcal{H} y^{\prime}-\mathcal{H} y_{N}^{\prime}\right) .
\end{aligned}
$$

The insertion of (29), (30) into (28) yields

$$
\begin{aligned}
e^{\prime}= & \mathcal{K}\left(y, y^{\prime}\right)-\mathcal{K}\left(y_{N}, y_{N}^{\prime}\right)+\Pi_{N}^{\theta, v} \mathcal{F} y-\Pi_{N}^{\theta, v} \mathcal{F} y_{N} \\
& +y^{\prime}-\Pi_{N}^{\theta, v} y^{\prime}+\Pi_{N}^{\theta, v}\left(\mathcal{K}\left(y, y^{\prime}\right)-\mathcal{K}\left(y_{N}, y_{N}^{\prime}\right)\right)-\left(\mathcal{K}\left(y, y^{\prime}\right)-\mathcal{K}\left(y_{N}, y_{N}^{\prime}\right)\right) \\
= & \mathcal{K}\left(y, y^{\prime}\right)-\mathcal{K}\left(y_{N}, y_{N}^{\prime}\right)+\Pi_{N}^{\theta, v} \mathcal{F} y-\Pi_{N}^{\theta, v} \mathcal{F} y_{N} \\
& +\Pi_{N}^{\theta, v}\left(\mathcal{K}\left(y, y^{\prime}\right)-\mathcal{K}\left(y_{N}, y_{N}^{\prime}\right)\right)-\left(\mathcal{K}\left(y, y^{\prime}\right)-\mathcal{K}\left(y_{N}, y_{N}^{\prime}\right)\right)+S_{1} .
\end{aligned}
$$




$$
e=\mathcal{H} e^{\prime}+y-\Pi_{N}^{\theta, v} y+\Pi_{N}^{\theta, v} \mathcal{H} e^{\prime}-\mathcal{H} e^{\prime}=\mathcal{H} e^{\prime}+S_{2}+S_{3}
$$

where

$$
S_{1}=y^{\prime}-\Pi_{N}^{\theta, v} y^{\prime}, \quad S_{2}=y-\Pi_{N}^{\theta, v} y, \quad S_{3}=\Pi_{N}^{\theta, v} \mathcal{H} e^{\prime}-\mathcal{H} e^{\prime},
$$

we use the Lagrange mean value differential theorem to deal with (31), there exists functions $\varphi(\sigma)=y_{N}(\sigma)+\lambda e(\sigma), \quad \psi(\sigma)=y_{N}^{\prime}(\sigma)+\vartheta e^{\prime}(\sigma), \quad \phi(\sigma)=y_{N}(\sigma)+\iota e(\sigma),(0<$ $\lambda, \vartheta, \iota<1)$ such that

$$
\begin{aligned}
\left(\mathcal{K}\left(y, y^{\prime}\right)\right)(z)-\left(\mathcal{K}\left(y_{N}, y_{N}^{\prime}\right)\right)(z)= & \int_{-1}^{z}(z-\sigma)^{-\mu}\left(\widetilde{K}\left(z, \sigma, y(\sigma), y^{\prime}(\sigma)\right)\right. \\
& \left.-\widetilde{K}\left(z, \sigma, y_{N}(\sigma), y_{N}^{\prime}(\sigma)\right)\right) d \sigma \\
= & \int_{-1}^{z}(z-\sigma)^{-\mu} \frac{\partial \widetilde{K}\left(z, \sigma, \varphi(\sigma), y^{\prime}(\sigma)\right)}{\partial \widetilde{K}_{3}} e(\sigma) d \sigma \\
& +\int_{-1}^{z}(z-\sigma)^{-\mu} \frac{\partial \widetilde{K}\left(z, \sigma, y_{N}(\sigma), \psi(\sigma)\right)}{\partial \widetilde{K}_{4}} e^{\prime}(\sigma) d \sigma \\
:= & \left(\mathcal{K}_{1} e\right)(z)+\left(\mathcal{K}_{2} e^{\prime}\right)(z) . \\
(\mathcal{F} y)(z)-\left(\mathcal{F} y_{N}\right)(z)= & f(z, y(z))-f\left(z, y_{N}(z)\right)=\frac{\partial f(z, \phi(z))}{\partial f_{2}} e(z) .
\end{aligned}
$$

where

$$
\begin{aligned}
\left(\mathcal{K}_{1} e\right)(z) & =\int_{-1}^{z}(z-\sigma)^{-\mu} \frac{\partial \widetilde{K}\left(z, \sigma, \varphi(\sigma), y^{\prime}(\sigma)\right)}{\partial \widetilde{K}_{3}} e(\sigma) d \sigma \\
\left(\mathcal{K}_{2} e^{\prime}\right)(z) & =\int_{-1}^{z}(z-\sigma)^{-\mu} \frac{\partial \widetilde{K}\left(z, \sigma, y_{N}(\sigma), \psi(\sigma)\right)}{\partial \widetilde{K}_{4}} e^{\prime}(\sigma) d \sigma .
\end{aligned}
$$

and $\frac{\partial \widetilde{K}}{\partial \widetilde{K}_{j}}(j=3,4)$ is denoted the partial derivatives of $\widetilde{K}$ for its $j$ th arguments. $\frac{\partial f}{\partial f_{2}}$ represents the partial derivative of $f$ for its second argument.

The insertion of (33), (34) and (32) into (31) yields

$$
\begin{aligned}
e^{\prime}(z)= & \left(\mathcal{K}_{1} e\right)(z)+\left(\mathcal{K}_{2} e^{\prime}\right)(z)+\Pi_{N}^{\theta, v}\left(\frac{\partial f(x, \phi(z))}{\partial f_{2}} e(z)\right) \\
& +\Pi_{N}^{\theta, v}\left(\mathcal{K}_{1} e\right)(z)-\left(\mathcal{K}_{1} e\right)(z)+\Pi_{N}^{\theta, v}\left(\mathcal{K}_{2} e^{\prime}\right)(z)-\left(\mathcal{K}_{2} e^{\prime}\right)(z)+S_{1}(z) \\
= & \int_{-1}^{z} \int_{-1}^{\sigma}(z-\sigma)^{-\mu} \frac{\partial \widetilde{K}\left(z, \sigma, \varphi(\sigma), y^{\prime}(\sigma)\right)}{\partial \widetilde{K}_{3}} e^{\prime}(s) d s d \sigma+\frac{\partial f(z, \phi(z))}{\partial f_{2}} \int_{-1}^{z} e^{\prime}(\sigma) d \sigma \\
& +\int_{-1}^{z}(z-\sigma)^{-\mu} \frac{\partial \widetilde{K}\left(z, \sigma, \varphi(\sigma), y^{\prime}(\sigma)\right)}{\partial \widetilde{K}_{3}}\left(S_{2}(\sigma)+S_{3}(\sigma)\right) d \sigma \\
& +\int_{-1}^{z}(z-\sigma)^{-\mu} \frac{\partial \widetilde{K}\left(z, \sigma, y_{N}(\sigma), \psi(\sigma)\right)}{\partial \widetilde{K}_{4}} e^{\prime}(\sigma) d \sigma+S_{1}(z)+\sum_{j=4}^{8} S_{j}(z) .
\end{aligned}
$$


where

$$
\begin{aligned}
& S_{4}(z)=\Pi_{N}^{\theta, v}\left(\mathcal{K}_{1} e\right)(z)-\left(\mathcal{K}_{1} e\right)(z), S_{5}(z)=\Pi_{N}^{\theta, v}\left(\mathcal{K}_{2} e^{\prime}\right)(z)-\left(\mathcal{K}_{2} e^{\prime}\right)(z), \\
& S_{6}(z)=\Pi_{N}^{\theta, v}\left(\frac{\partial f(z, \phi(z))}{\partial f_{2}} S_{2}(z)\right), S_{7}(z)=\Pi_{N}^{\theta, v}\left(\frac{\partial f(z, \phi(z))}{\partial f_{2}} S_{3}(z)\right), \\
& S_{8}(z)=\Pi_{N}^{\theta, v}\left(\frac{\partial f(z, \phi(z))}{\partial f_{2}} \int_{-1}^{z} e^{\prime}(\sigma) d \sigma\right)-\frac{\partial f(z, \phi(z))}{\partial f_{2}} \int_{-1}^{z} e^{\prime}(\sigma) d \sigma .
\end{aligned}
$$

By the Dirichlet formula, namely $\int_{-1}^{z} \int_{-1}^{\sigma} \phi(\sigma, s) d s d \sigma=\int_{-1}^{z} \int_{s}^{z} \phi(\sigma, s) d \sigma d s$, we have

$$
\begin{aligned}
e^{\prime}(z)= & \int_{-1}^{z}\left(\frac{\partial f(z, \phi(z))}{\partial f_{2}}+\int_{s}^{z}(z-\sigma)^{-\mu} \frac{\partial \widetilde{K}\left(z, \sigma, \varphi(\sigma), y^{\prime}(\sigma)\right)}{\partial \widetilde{K}_{3}} d \sigma\right) e^{\prime}(s) d s \\
& +\int_{-1}^{z}(z-\sigma)^{-\mu} \frac{\partial \widetilde{K}\left(z, \sigma, y_{N}(\sigma), \psi(\sigma)\right)}{\partial \widetilde{K}_{4}} e^{\prime}(\sigma) d \sigma+\mathcal{S}(z) .
\end{aligned}
$$

where

$$
\mathcal{S}(z)=\int_{-1}^{z}(z-\sigma)^{-\mu} \frac{\partial \widetilde{K}\left(z, \sigma, \varphi(\sigma), y^{\prime}(\sigma)\right)}{\partial \widetilde{K}_{3}}\left(S_{2}(\sigma)+S_{3}(\sigma)\right) d \sigma+S_{1}(z)+\sum_{j=4}^{8} S_{j}(z) .
$$

Set $\mathcal{D}_{1}=\{(z, \sigma):-1 \leq \sigma \leq z,-1 \leq z \leq 1\}$, we have

$$
\begin{aligned}
& \left|\frac{\partial f(z, \phi(z))}{\partial f_{2}}+\int_{s}^{z}(z-\sigma)^{-\mu} \frac{\partial \widetilde{K}\left(z, \sigma, \varphi(\sigma), y^{\prime}(\sigma)\right)}{\partial \widetilde{K}_{3}} d \sigma\right| \\
& \leq \max _{z \in[-1,1]}\left|\frac{\partial f(z, \phi(z))}{\partial f_{2}}\right|+\max _{(z, \sigma) \in \mathcal{D}_{1}}\left|\frac{\partial \widetilde{K}\left(z, \sigma, \varphi(\sigma), y^{\prime}(\sigma)\right)}{\partial \widetilde{K}_{3}}\right| \int_{s}^{z}(z-\sigma)^{-\mu} d \sigma \\
& \leq \max _{z \in[-1,1]}\left|\frac{\partial f(z, \phi(z))}{\partial f_{2}}\right|+\frac{2^{1-\mu}}{1-\mu} \max _{(z, \sigma) \in \mathcal{D}_{1}}\left|\frac{\partial \widetilde{K}\left(z, \sigma, \varphi(\sigma), y^{\prime}(\sigma)\right)}{\partial \widetilde{K}_{3}}\right| \equiv M_{1} \\
& \left|\frac{\partial \widetilde{K}\left(z, \sigma, y_{N}(\sigma), \psi(\sigma)\right)}{\partial \widetilde{K}_{4}}\right| \leq \max _{(z, \sigma) \in \mathcal{D}_{1}}\left|\frac{\partial \widetilde{K}\left(z, \sigma, y_{N}(\sigma), \psi(\sigma)\right)}{\partial \widetilde{K}_{4}}\right| \equiv M_{2}
\end{aligned}
$$

Then, from the above formula, (36) can be written as

$$
\left|e^{\prime}(z)\right| \leq M_{1} \int_{-1}^{z}\left|e^{\prime}(\sigma)\right| d \sigma+M_{2} \int_{-1}^{z}(z-\sigma)^{-\mu}\left|e^{\prime}(\sigma)\right| d \sigma+|\mathcal{S}(z)|
$$

By using (32), (37) and Lemma 3.6, we have

$$
\begin{aligned}
& \left\|e^{\prime}\right\|_{\infty} \leq C\|\mathcal{S}\|_{\infty} \leq C \sum_{i=1}^{8}\left\|S_{i}\right\|_{\infty}, \\
& \|e\|_{\infty} \leq C\left\|e^{\prime}\right\|_{\infty}+\left\|S_{2}\right\|_{\infty}+\left\|S_{3}\right\|_{\infty} \leq C \sum_{i=1}^{8}\left\|S_{i}\right\|_{\infty} .
\end{aligned}
$$

Due to Lemma 3.1, we have

$$
\begin{aligned}
& \left\|S_{1}\right\|_{\infty}=\left\|y^{\prime}-\Pi_{N}^{\theta, \nu} y^{\prime}\right\|_{\infty} \leq C N^{\frac{3}{4}-m}\left|y^{\prime}\right|_{H_{\omega \theta}^{m ; \nu}}^{m ; N}, \\
& \left\|S_{2}\right\|_{\infty}=\left\|y-\Pi_{N}^{\theta, v} y\right\|_{\infty} \leq C N^{\frac{3}{4}-m}|y|_{H_{\omega^{\theta, v}}^{m ; N}} .
\end{aligned}
$$


In the virtue of Lemma 3.1 with $m=1$, we obtain

$$
\begin{aligned}
\left\|S_{3}\right\|_{\infty} & =\left\|\Pi_{N}^{\theta, v} \mathcal{H} e^{\prime}-\mathcal{H} e^{\prime}\right\|_{\infty} \leq C N^{-\frac{1}{4}}\left|\mathcal{H} e^{\prime}\right|_{H_{\omega}^{1 ; N}} \underset{\omega^{\theta, v}}{ } \\
& \leq C N^{-\frac{1}{4}}\left\|e^{\prime}\right\|_{\omega^{\theta, v}} \leq C N^{-\frac{1}{4}}\left\|e^{\prime}\right\|_{\infty} .
\end{aligned}
$$

Furthermore, in the virtue of Lemma 3.1 with $m=1$ and Lemma 3.8, we have

$$
\begin{aligned}
\left\|S_{8}\right\|_{\infty} & \leq C N^{-\frac{1}{4}}\left|\frac{\partial f(z, \phi(z))}{\partial f_{2}} \int_{-1}^{z} e^{\prime}(\sigma) d \sigma\right|_{H_{\omega^{\theta, v}}^{1 ; N}} \\
& \leq C N^{-\frac{1}{4}}\left\|e^{\prime}\right\|_{\omega^{\theta, v}} \leq C N^{-\frac{1}{4}}\left\|e^{\prime}\right\|_{\infty} .
\end{aligned}
$$

Next, by applying the (22), Lemma 3.7 and Lemma 3.9, we get

$$
\begin{aligned}
\left\|S_{4}\right\|_{\infty} & =\left\|\Pi_{N}^{\theta, v} \mathcal{K}_{1} e-\mathcal{K}_{1} e\right\|_{\infty}=\left\|\left(\Pi_{N}^{\theta, v}-I\right)\left(\mathcal{K}_{1} e-\mathfrak{L}_{N} \mathcal{K}_{1} e\right)\right\|_{\infty} \\
& \leq\left\|\Pi_{N}^{\theta, v}\left(\mathcal{K}_{1} e-\mathfrak{L}_{N} \mathcal{K}_{1} e\right)\right\|_{\infty}+\left\|\mathcal{K}_{1} e-\mathfrak{L}_{N} \mathcal{K}_{1} e\right\|_{\infty} \\
& \leq C\left\|\mathcal{K}_{1} e-\mathfrak{L}_{N} \mathcal{K}_{1} e\right\|_{\infty} \leq C N^{-\varrho}\left\|\mathcal{K}_{1} e\right\|_{0, \varrho} \leq C N^{-\varrho}\|e\|_{\infty} \\
& \leq C N^{-\varrho}\left(\left\|e^{\prime}\right\|_{\infty}+\left\|S_{2}\right\|_{\infty}+\left\|S_{3}\right\|_{\infty}\right) . \\
\left\|S_{5}\right\|_{\infty} & =\left\|\Pi_{N}^{\theta, v} \mathcal{K}_{2} e^{\prime}-\mathcal{K}_{2} e^{\prime}\right\|_{\infty}=\left\|\left(\Pi_{N}^{\theta, v}-I\right)\left(\mathcal{K}_{2} e^{\prime}-\mathfrak{L}_{N} \mathcal{K}_{2} e^{\prime}\right)\right\|_{\infty} \\
& \leq\left\|\Pi_{N}^{\theta, v}\left(\mathcal{K}_{2} e^{\prime}-\mathfrak{L}_{N} \mathcal{K}_{2} e^{\prime}\right)\right\|_{\infty}+\left\|\mathcal{K}_{2} e^{\prime}-\mathfrak{L}_{N} \mathcal{K}_{2} e^{\prime}\right\|_{\infty} \\
& \leq C\left\|\mathcal{K}_{2} e^{\prime}-\mathfrak{L}_{N} \mathcal{K}_{2} e^{\prime}\right\|_{\infty} \leq C N^{-\varrho}\left\|\mathcal{K}_{2} e^{\prime}\right\|_{0, \varrho} \leq C N^{-\varrho}\left\|e^{\prime}\right\|_{\infty} .
\end{aligned}
$$

where $0<\varrho<1-\mu$.

Then, due to Lemma 3.9, (39) and (40) yields

$$
\begin{aligned}
& \left\|S_{6}\right\|_{\infty}=\left\|\Pi_{N}^{\theta, v}\left(\frac{\partial f(z, \phi(z))}{\partial f_{2}} S_{2}(z)\right)\right\|_{\infty} \leq C\left\|\frac{\partial f(z, \phi(z))}{\partial f_{2}} S_{2}(z)\right\|_{\infty} \leq C N^{\frac{3}{4}-m}|y|_{H_{\omega^{\theta, v}}^{m ; N},}, \\
& \left\|S_{7}\right\|_{\infty}=\left\|\Pi_{N}^{\theta, v}\left(\frac{\partial f(z, \phi(z))}{\partial f_{2}} S_{3}(z)\right)\right\|_{\infty} \leq C\left\|\frac{\partial f(z, \phi(z))}{\partial f_{2}} S_{3}(z)\right\|_{\infty} \leq C N^{-\frac{1}{4}}\left\|e^{\prime}\right\|_{\infty} .
\end{aligned}
$$

On the other hand, by using (32), (37), Lemma 3.6 and Lemma 3.8, we have

$$
\begin{aligned}
& \left\|e^{\prime}\right\|_{\omega^{\theta, v}} \leq C\|\mathcal{S}\|_{\omega^{\theta, v}} \leq C \sum_{i=1}^{8}\left\|S_{i}\right\|_{\omega^{\theta, v}}, \\
& \|e\|_{\omega^{\theta, \nu}} \leq C\left\|e^{\prime}\right\|_{\omega^{\theta, \nu}}+\left\|S_{2}\right\|_{\omega^{\theta, \nu}}+\left\|S_{3}\right\|_{\omega^{\theta, \nu}} \leq C \sum_{i=1}^{8}\left\|S_{i}\right\|_{\omega^{\theta, v}} .
\end{aligned}
$$

Due to Lemma 3.1, we have

$$
\begin{aligned}
& \left\|S_{1}\right\|_{\omega^{\theta, v}}=\left\|y^{\prime}-\Pi_{N}^{\theta, v} y^{\prime}\right\|_{\omega^{\theta, v}} \leq C N^{-m}\left|y^{\prime}\right|_{H_{\omega^{\theta, v}}^{m ; N}}, \\
& \left\|S_{2}\right\|_{\omega^{\theta, v}}=\left\|y-\Pi_{N}^{\theta, v} y\right\|_{\omega^{\theta, v}} \leq C N^{-m}|y|_{\substack{H_{\omega^{\theta, v}}^{m ; N} \\
\text { m. }}} \leq
\end{aligned}
$$

By using Lemma 3.1 with $m=1$, we obtain

$$
\left\|S_{3}\right\|_{\omega^{\theta, v}}=\left\|\Pi_{N}^{\theta, \nu} \mathcal{H} e^{\prime}-\mathcal{H} e^{\prime}\right\|_{\omega^{\theta, v}} \leq C N^{-1}\left|\mathcal{H} e^{\prime}\right|_{H_{\omega^{\theta, v}}^{1 ; N}} \leq C N^{-1}\left\|e^{\prime}\right\|_{\omega^{\theta, v}} .
$$


In addition, we use Lemma 3.8 and Lemma 3.1 with $m=1$, yields

$$
\left\|S_{8}\right\|_{\omega^{\theta, \nu}} \leq C N^{-1}\left|\frac{\partial f(z, \phi(z))}{\partial f_{2}} \int_{-1}^{z} e^{\prime}(\sigma) d \sigma\right|_{H_{\omega^{\theta, \nu}}^{1 ; N}} \leq C N^{-1}\left\|e^{\prime}\right\|_{\omega^{\theta, v}} .
$$

Moreover, it is similar to the derivation process of (42) and (43). Applying (22), Lemma 3.7 and Lemma 3.9, we have

$$
\begin{aligned}
\left\|S_{4}\right\|_{\omega^{\theta, v}} & =\left\|\Pi_{N}^{\theta, v} \mathcal{K}_{1} e-\mathcal{K}_{1} e\right\|_{\omega^{\theta, v}}=\left\|\left(\Pi_{N}^{\theta, v}-I\right)\left(\mathcal{K}_{1} e-\mathfrak{L}_{N} \mathcal{K}_{1} e\right)\right\|_{\omega^{\theta, v}} \\
& \leq\left\|\Pi_{N}^{\theta, v}\left(\mathcal{K}_{1} e-\mathfrak{L}_{N} \mathcal{K}_{1} e\right)\right\|_{\omega^{\theta, v}}+\left\|\mathcal{K}_{1} e-\mathfrak{L}_{N} \mathcal{K}_{1} e\right\|_{\omega^{\theta, v}} \\
& \leq C\left\|\mathcal{K}_{1} e-\mathfrak{L}_{N} \mathcal{K}_{1} e\right\|_{\omega^{\theta, v}} \leq C\left\|\mathcal{K}_{1} e-\mathfrak{L}_{N} \mathcal{K}_{1} e\right\|_{\infty} \\
& \leq C N^{-\varrho}\left\|\mathcal{K}_{1} e\right\|_{0, \varrho} \leq C N^{-\varrho}\|e\|_{\infty} . \\
\left\|S_{5}\right\|_{\omega^{\theta, v}} & =\left\|\Pi_{N}^{\theta, v} \mathcal{K}_{2} e^{\prime}-\mathcal{K}_{2} e^{\prime}\right\|_{\omega^{\theta, v}}=\left\|\left(\Pi_{N}^{\theta, v}-I\right)\left(\mathcal{K}_{2} e^{\prime}-\mathfrak{L}_{N} \mathcal{K}_{2} e^{\prime}\right)\right\|_{\omega^{\theta, v}} \\
& \leq\left\|\Pi_{N}^{\theta, v}\left(\mathcal{K}_{2} e^{\prime}-\mathfrak{L}_{N} \mathcal{K}_{2} e^{\prime}\right)\right\|_{\omega^{\theta, v}}+\left\|\mathcal{K}_{2} e^{\prime}-\mathfrak{L}_{N} \mathcal{K}_{2} e^{\prime}\right\|_{\omega^{\theta, v}} \\
& \leq C\left\|\mathcal{K}_{2} e^{\prime}-\mathfrak{L}_{N} \mathcal{K}_{2} e^{\prime}\right\|_{\omega^{\theta, v}} \leq C\left\|\mathcal{K}_{2} e^{\prime}-\mathfrak{L}_{N} \mathcal{K}_{2} e^{\prime}\right\|_{\infty} \\
& \leq C N^{-\varrho}\left\|\mathcal{K}_{2} e^{\prime}\right\|_{0, \varrho} \leq C N^{-\varrho}\left\|e^{\prime}\right\|_{\infty} .
\end{aligned}
$$

where $0<\varrho<1-\mu$.

Then, by employing (46), (47) and Lemma 3.9 yields

$$
\begin{aligned}
& \left\|S_{6}\right\|_{\omega^{\theta, v}} \leq C\left\|\frac{\partial f(z, \phi(z))}{\partial f_{2}} S_{2}(z)\right\|_{\omega^{\theta, v}} \leq C\left\|S_{2}\right\|_{\omega^{\theta, v}} \leq C N^{-m}|y|_{H_{\omega^{\theta, v}}^{m ; N}} . \\
& \left\|S_{7}\right\|_{\omega^{\theta, v}} \leq C\left\|\frac{\partial f(z, \phi(z))}{\partial f_{2}} S_{3}(z)\right\|_{\omega^{\theta, v}} \leq C\left\|S_{3}\right\|_{\omega^{\theta, v}} \leq C N^{-1}\left\|e^{\prime}\right\|_{\omega^{\theta, v}} .
\end{aligned}
$$

Therefore, when the provided $N$ is large enough, we obtain the desired estimate (26), (27).

\subsection{Jacobi pseudo-spectral Galerkin method}

For this subsection, according to the definition of the interpolation operator $I_{N}^{\theta, v}$ and (12), the pseudo-spectral Galerkin approximate solution $\bar{y}_{N}, \bar{y}_{N}^{\prime}$ satisfies

$$
\left\{\begin{array}{l}
\left(\bar{y}_{N}^{\prime}, \psi_{N}\right)_{\omega^{\theta, v}}=\left(I_{N}^{\theta, v} \mathcal{F} \bar{y}_{N}, \psi_{N}\right)_{\omega^{\theta, v}}+\left(I_{N}^{\theta, v} \mathcal{K}_{N}\left(\bar{y}_{N}, \bar{y}_{N}^{\prime}\right), \psi_{N}\right)_{\omega^{\theta, v}}, \quad \forall \psi_{N} \in \mathcal{P}_{N} . \\
\left(\bar{y}_{N}, \psi_{N}\right)_{\omega^{\theta, v}}=\left(I_{N}^{\theta, v} y_{-1}, \psi_{N}\right)_{\omega^{\theta, v}}+\left(I_{N}^{\theta, v} \mathcal{H}_{N} \bar{y}_{N}^{\prime}, \psi_{N}\right)_{\omega^{\theta, v}},
\end{array}\right.
$$

Let

$$
\begin{aligned}
L(z)= & \left(\mathcal{K}\left(\bar{y}_{N}, \bar{y}_{N}^{\prime}\right)\right)(z)-\left(\mathcal{K}_{N}\left(\bar{y}_{N}, \bar{y}_{N}^{\prime}\right)\right)(z) \\
= & \left(\frac{1+z}{2}\right)^{1-\mu} \int_{-1}^{1}(1-\zeta)^{-\mu} \widetilde{K}\left(z, \sigma(z, \zeta), \bar{y}_{N}(\sigma(z, \zeta)), \bar{y}_{N}^{\prime}(\sigma(z, \zeta))\right) d \zeta \\
& -\left(\frac{1+z}{2}\right)^{1-\mu} \sum_{i=0}^{N} \widetilde{K}\left(z, \sigma\left(z, \zeta_{i}\right), \bar{y}_{N}\left(\sigma\left(z, \zeta_{i}\right)\right), \bar{y}_{N}^{\prime}\left(\sigma\left(z, \zeta_{i}\right)\right)\right) \omega_{i}^{-\mu, 0}
\end{aligned}
$$


Note that $\bar{y}_{N}^{\prime}(z) \in \mathcal{P}_{N}$, then

$$
\begin{aligned}
\left(1, \bar{y}_{N}^{\prime}(\sigma(z, \zeta))\right)_{\omega^{0,0}} & =\int_{-1}^{1} \bar{y}_{N}^{\prime}(\sigma(z, \zeta)) d \zeta=\sum_{j=0}^{N} \bar{y}_{N}^{\prime}(\sigma(z, \zeta j)) \omega_{j}^{0,0} \\
& =\left(1, \bar{y}_{N}^{\prime}(\sigma(z, \zeta))\right)_{\omega^{0,0}, N}
\end{aligned}
$$

Thus, we have $\mathcal{H} \bar{y}_{N}^{\prime}=\mathcal{H}_{N} \bar{y}_{N}^{\prime}$. And then, the combination of (53), (54) and (55), yields

$$
\left\{\begin{array}{l}
\left(\bar{y}_{N}^{\prime}, \psi_{N}\right)_{\omega^{\theta, v}}=\left(I_{N}^{\theta, v} \mathcal{F} \bar{y}_{N}, \psi_{N}\right)_{\omega^{\theta, v}}+\left(I_{N}^{\theta, v} \mathcal{K}\left(\bar{y}_{N}, \bar{y}_{N}^{\prime}\right)-I_{N}^{\theta, v} L(z), \psi_{N}\right)_{\omega^{\theta, v}}, \\
\left(\bar{y}_{N}, \psi_{N}\right)_{\omega^{\theta, v}}=\left(I_{N}^{\theta, v} y_{-1}, \psi_{N}\right)_{\omega^{\theta, v}}+\left(I_{N}^{\theta, v} \mathcal{H} \bar{y}_{N}^{\prime}, \psi_{N}\right)_{\omega^{\theta, v}}
\end{array}\right.
$$

which gives rise to

$$
\left\{\begin{array}{l}
\bar{y}_{N}^{\prime}=I_{N}^{\theta, v} \mathcal{F} \bar{y}_{N}+I_{N}^{\theta, v} \mathcal{K}\left(\bar{y}_{N}, \bar{y}_{N}^{\prime}\right)-I_{N}^{\theta, v} L(z) \\
\bar{y}_{N}=y_{-1}+I_{N}^{\theta, v} \mathcal{H} \bar{y}_{N}^{\prime} .
\end{array}\right.
$$

By the discussion above, (53) (56) and (57) are equivalent.

Theorem 4.2 Assume that $\bar{y}_{N}(z), \bar{y}_{N}^{\prime}(z)$ is the Jacobi pseudo-spectral Galerkin approximate solution and approximate derivative of (6), which is decided by (12). If the exact solution $y(z) \in H_{\omega^{\theta, v}}^{m+1 ; N}(\Lambda) \cap H_{\omega^{c}}^{m+1 ; N}(\Lambda)(m \geq 1)$ and $\widetilde{K}$ fulfills $m$ times Lipschitz continuous conditions for its third and fourth arguments, namely

$$
\left|\frac{\partial^{p} \widetilde{K}\left(z, \sigma, u_{1}(\sigma), u_{2}(\sigma)\right)}{\partial \sigma^{p}}-\frac{\partial^{p} \widetilde{K}\left(z, \sigma, v_{1}(\sigma), v_{2}(\sigma)\right)}{\partial \sigma^{p}}\right| \leq \sum_{q=1}^{2} L_{q p}\left|u_{q}(\sigma)-v_{q}(\sigma)\right|
$$

then the following error estimates hold

$$
\begin{aligned}
& \left\|y^{(i)}-\bar{y}_{N}^{(i)}\right\|_{\infty} \leq\left\{\begin{array}{l}
C N^{\frac{1}{2}-m} \log N \mathcal{V}_{1}, \quad-1<\theta, v \leq-\frac{1}{2}, \\
C N^{\gamma+1-m} \mathcal{V}_{2}, \quad-\frac{1}{2}<\gamma<\min \left\{\frac{1}{2}-\mu,-\frac{1}{4}\right\} .
\end{array}\right. \\
& \left\|y^{(i)}-\bar{y}_{N}^{(i)}\right\|_{\omega^{\theta, v}} \\
& \leq\left\{\begin{array}{l}
C N^{-m}\left(\mathcal{U}+\mathcal{K}^{*}+N^{\frac{1}{2}} \log N|y|_{H_{\omega^{c}}^{m ; N}}+\left(N^{\frac{1}{2}-\varrho} \log N+\log ^{2} N\right) \mathcal{V}_{1}\right), \quad-1<\theta, v \leq-\frac{1}{2}, \\
C N^{-m}\left(\mathcal{U}+\mathcal{K}^{*}+N^{\gamma+1}|y|_{H^{m}}^{m: N}+\left(N^{\gamma+1-\varrho}+N^{2 \gamma+1}\right) \mathcal{V}_{2}\right), \quad-\frac{1}{2}<\gamma<\min \left\{\frac{1}{2}-\mu,-\frac{1}{4}\right\} .
\end{array}\right.
\end{aligned}
$$

where

$$
\begin{aligned}
& \mathcal{K}^{*}=\max _{z \in[-1,1]}\left|\widetilde{K}\left(z, \sigma, y(\sigma), y^{\prime}(\sigma)\right)\right|_{H_{\omega^{-\mu, 0}}^{m ; N}}, \quad i=0,1, \quad p=0,1, \ldots, m, \\
& \max \left\{\frac{1}{2}+\gamma, 0\right\}<\varrho<1-\mu, \quad \gamma=\max \{\theta, \nu\}<\min \left\{\frac{1}{2}-\mu,-\frac{1}{4}\right\}, \\
& \mathcal{V}_{1}:=\log N|y|_{H_{\omega^{c}}^{m ; N}}+\left|y^{\prime}\right|_{H_{\omega^{c}}^{m ; N}}+N^{-\frac{1}{2}} \mathcal{K}^{*}, \quad \mathcal{V}_{2}:=N^{\gamma+\frac{1}{2}}|y|_{H_{\omega^{c}}^{m ; N}}+\left|y^{\prime}\right|_{H_{\omega^{c}}^{m ; N}}+N^{-\frac{1}{2}} \mathcal{K}^{*} .
\end{aligned}
$$

Proof Set $\varepsilon=y-\bar{y}_{N}, \quad \varepsilon^{\prime}=y^{\prime}-\bar{y}_{N}^{\prime}$. 
Subtraction (57) from (6), we have

$$
\left\{\begin{array}{l}
y^{\prime}-\bar{y}_{N}^{\prime}=\mathcal{F} y-I_{N}^{\theta, v} \mathcal{F} \bar{y}_{N}+\mathcal{K}\left(y, y^{\prime}\right)-I_{N}^{\theta, v} \mathcal{K}\left(\bar{y}_{N}, \bar{y}_{N}^{\prime}\right)+I_{N}^{\theta, v} L(z), \\
y-\bar{y}_{N}=\mathcal{H} y^{\prime}-I_{N}^{\theta, v} \mathcal{H} \bar{y}_{N}^{\prime} .
\end{array}\right.
$$

Direct computations show that

$$
\begin{gathered}
\mathcal{K}\left(y, y^{\prime}\right)-I_{N}^{\theta, v} \mathcal{K}\left(\bar{y}_{N}, \bar{y}_{N}^{\prime}\right)=y^{\prime}-\mathcal{F} y-I_{N}^{\theta, v}\left(y^{\prime}-\mathcal{F} y\right)+\left(\mathcal{K}\left(y, y^{\prime}\right)-\mathcal{K}\left(\bar{y}_{N}, \bar{y}_{N}^{\prime}\right)\right) \\
+I_{N}^{\theta, v} \mathcal{K}\left(y, y^{\prime}\right)-I_{N}^{\theta, v} \mathcal{K}\left(\bar{y}_{N}, \bar{y}_{N}^{\prime}\right)-\left(\mathcal{K}\left(y, y^{\prime}\right)-\mathcal{K}\left(\bar{y}_{N}, \bar{y}_{N}^{\prime}\right)\right) . \\
\mathcal{H} y^{\prime}-I_{N}^{\theta, v} \mathcal{H} \bar{y}_{N}^{\prime}=y-I_{N}^{\theta, v} y+\mathcal{H} y^{\prime}-\mathcal{H} \bar{y}_{N}^{\prime} \\
+I_{N}^{\theta, v}\left(\mathcal{H} y^{\prime}-\mathcal{H} \bar{y}_{N}^{\prime}\right)-\left(\mathcal{H} y^{\prime}-\mathcal{H} \bar{y}_{N}^{\prime}\right) .
\end{gathered}
$$

The insertion of (61),(62) into (60) yields

$$
\begin{aligned}
\varepsilon^{\prime}= & \mathcal{K}\left(y, y^{\prime}\right)-\mathcal{K}\left(\bar{y}_{N}, \bar{y}_{N}^{\prime}\right)+I_{N}^{\theta, v} \mathcal{F} y-I_{N}^{\theta, v} \mathcal{F} \bar{y}_{N}+y^{\prime}-I_{N}^{\theta, v} y^{\prime} \\
& +I_{N}^{\theta, v}\left(\mathcal{K}\left(y, y^{\prime}\right)-\mathcal{K}\left(\bar{y}_{N}, \bar{y}_{N}^{\prime}\right)\right)-\left(\mathcal{K}\left(y, y^{\prime}\right)-\mathcal{K}\left(\bar{y}_{N}, \bar{y}_{N}^{\prime}\right)\right)+I_{N}^{\theta, v} L(z) \\
= & \mathcal{K}\left(y, y^{\prime}\right)-\mathcal{K}\left(\bar{y}_{N}, \bar{y}_{N}^{\prime}\right)+I_{N}^{\theta, v} \mathcal{F} y-I_{N}^{\theta, v} \mathcal{F} \bar{y}_{N} \\
& +I_{N}^{\theta, v}\left(\mathcal{K}\left(y, y^{\prime}\right)-\mathcal{K}\left(\bar{y}_{N}, \bar{y}_{N}^{\prime}\right)\right)-\left(\mathcal{K}\left(y, y^{\prime}\right)-\mathcal{K}\left(\bar{y}_{N}, \bar{y}_{N}^{\prime}\right)\right)+Q_{1}+I_{N}^{\theta, v} L(z) . \\
\varepsilon= & \mathcal{H} y^{\prime}-\mathcal{H} \bar{y}_{N}^{\prime}+y-I_{N}^{\theta, v} y+I_{N}^{\theta, v}\left(\mathcal{H} y^{\prime}-\mathcal{H} \bar{y}_{N}^{\prime}\right)-\left(\mathcal{H} y^{\prime}-\mathcal{H} \bar{y}_{N}^{\prime}\right) \\
= & \mathcal{H} \varepsilon^{\prime}+y-I_{N}^{\theta, v} y+I_{N}^{\theta, v} \mathcal{H} \varepsilon^{\prime}-\mathcal{H} \varepsilon^{\prime} \\
= & \mathcal{H} \varepsilon^{\prime}+Q_{2}+Q_{3} .
\end{aligned}
$$

where

$$
Q_{1}=y^{\prime}-I_{N}^{\theta, v} y^{\prime}, \quad Q_{2}=y-I_{N}^{\theta, v} y, \quad Q_{3}=I_{N}^{\theta, v} \mathcal{H} \varepsilon^{\prime}-\mathcal{H} \varepsilon^{\prime} .
$$

By using the Lagrange mean value differential theorem to (63), there exists functions $\bar{\varphi}(\sigma)=\bar{y}_{N}(\sigma)+\bar{\lambda} \varepsilon(\sigma), \quad \bar{\psi}(\sigma)=\bar{y}_{N}^{\prime}(\sigma)+\bar{\vartheta} \varepsilon^{\prime}(\sigma), \quad \bar{\phi}(\sigma)=\bar{y}_{N}(\sigma)+\bar{\imath} \varepsilon(\sigma), \quad(0<$ $\bar{\lambda}, \bar{\vartheta}, \bar{\imath}<1)$ such that

$$
\begin{aligned}
\left(\mathcal{K}\left(y, y^{\prime}\right)\right)(z)-\left(\mathcal{K}\left(\bar{y}_{N}, \bar{y}_{N}^{\prime}\right)\right)(z)= & \int_{-1}^{z}(z-\sigma)^{-\mu}\left(\widetilde{K}\left(z, \sigma, y(\sigma), y^{\prime}(\sigma)\right)\right. \\
& \left.-\widetilde{K}\left(z, \sigma, \bar{y}_{N}(\sigma), \bar{y}_{N}^{\prime}(\sigma)\right)\right) d \sigma \\
= & \int_{-1}^{z}(z-\sigma)^{-\mu} \frac{\partial \widetilde{K}\left(z, \sigma, \bar{\varphi}(\sigma), y^{\prime}(\sigma)\right)}{\partial \widetilde{K}_{3}} \varepsilon(\sigma) d \sigma \\
& +\int_{-1}^{z}(z-\sigma)^{-\mu} \frac{\partial \widetilde{K}\left(z, \sigma, \bar{y}_{N}(\sigma), \bar{\psi}(\sigma)\right)}{\partial \widetilde{K}_{4}} \varepsilon^{\prime}(\sigma) d \sigma \\
:= & \left(\mathcal{K}_{3} \varepsilon\right)(z)+\left(\mathcal{K}_{4} \varepsilon^{\prime}\right)(z) .
\end{aligned}
$$

$$
(\mathcal{F} y)(z)-\left(\mathcal{F} \bar{y}_{N}\right)(z)=f(z, y(z))-f\left(z, \bar{y}_{N}(z)\right)=\frac{\partial f(z, \bar{\phi}(z))}{\partial f_{2}} \varepsilon(z) .
$$

where

$$
\begin{aligned}
\left(\mathcal{K}_{3} \varepsilon\right)(z) & =\int_{-1}^{z}(z-\sigma)^{-\mu} \frac{\partial \widetilde{K}\left(z, \sigma, \bar{\varphi}(\sigma), y^{\prime}(\sigma)\right)}{\partial \widetilde{K}_{3}} \varepsilon(\sigma) d \sigma \\
\left(\mathcal{K}_{4} \varepsilon^{\prime}\right)(z) & =\int_{-1}^{z}(z-\sigma)^{-\mu} \frac{\partial \widetilde{K}\left(z, \sigma, \bar{y}_{N}(\sigma), \bar{\psi}(\sigma)\right)}{\partial \widetilde{K}_{4}} \varepsilon^{\prime}(\sigma) d \sigma .
\end{aligned}
$$


and $\frac{\partial \widetilde{K}}{\partial \widetilde{K}_{j}}(j=3,4)$ is denoted the partial derivatives of $\widetilde{K}$ for its $j$ th arguments. $\frac{\partial f}{\partial f_{2}}$ represents the partial derivative of $f$ for its second argument.

The insertion of (64), (65) and (66) into (63) yields

$$
\begin{aligned}
\varepsilon^{\prime}(z)= & \left(\mathcal{K}_{3} \varepsilon\right)(z)+\left(\mathcal{K}_{4} \varepsilon^{\prime}\right)(z)+I_{N}^{\theta, v}\left(\frac{\partial f(z, \bar{\phi}(z))}{\partial f_{2}} \varepsilon(z)\right) \\
& +I_{N}^{\theta, v}\left(\mathcal{K}_{3} \varepsilon\right)(z)-\left(\mathcal{K}_{3} \varepsilon\right)(z)+I_{N}^{\theta, v}\left(\mathcal{K}_{4} \varepsilon^{\prime}\right)(z)-\left(\mathcal{K}_{4} \varepsilon^{\prime}\right)(z)+Q_{1}(z)+I_{N}^{\theta, v} L(z) \\
= & \int_{-1}^{z} \int_{-1}^{\sigma}(z-\sigma)^{-\mu} \frac{\partial \widetilde{K}\left(z, \sigma, \bar{\varphi}(\sigma), y^{\prime}(\sigma)\right)}{\partial \widetilde{K}_{3}} \varepsilon^{\prime}(s) d s d \sigma+\frac{\partial f(z, \bar{\phi}(z))}{\partial f_{2}} \int_{-1}^{z} \varepsilon^{\prime}(\sigma) d \sigma \\
& +\int_{-1}^{z}(z-\sigma)^{-\mu} \frac{\partial \widetilde{K}\left(z, \sigma, \bar{\varphi}(\sigma), y^{\prime}(\sigma)\right)}{\partial \widetilde{K}_{3}}\left(Q_{2}(\sigma)+Q_{3}(\sigma)\right) d \sigma \\
& +\int_{-1}^{z}(z-\sigma)^{-\mu} \frac{\partial \widetilde{K}\left(z, \sigma, \bar{y}_{N}(\sigma), \bar{\psi}(\sigma)\right)}{\partial \widetilde{K}_{4}} \varepsilon^{\prime}(\sigma) d \sigma+Q_{1}(z)+\sum_{j=4}^{8} Q_{j}(z)+I_{N}^{\theta, v} L(z) .
\end{aligned}
$$

where

$$
\begin{aligned}
& Q_{4}(z)=I_{N}^{\theta, v}\left(\mathcal{K}_{3} \varepsilon\right)(z)-\left(\mathcal{K}_{3} \varepsilon\right)(z), Q_{5}(z)=I_{N}^{\theta, v}\left(\mathcal{K}_{4} \varepsilon^{\prime}\right)(z)-\left(\mathcal{K}_{4} \varepsilon^{\prime}\right)(z), \\
& Q_{6}(z)=I_{N}^{\theta, v}\left(\frac{\partial f(z, \bar{\phi}(z))}{\partial f_{2}} Q_{2}(z)\right), Q_{7}(z)=I_{N}^{\theta, v}\left(\frac{\partial f(z, \bar{\phi}(z))}{\partial f_{2}} Q_{3}(z)\right), \\
& Q_{8}(z)=I_{N}^{\theta, v}\left(\frac{\partial f(z, \bar{\phi}(z))}{\partial f_{2}} \int_{-1}^{z} \varepsilon^{\prime}(\sigma) d \sigma\right)-\frac{\partial f(z, \bar{\phi}(z))}{\partial f_{2}} \int_{-1}^{z} \varepsilon^{\prime}(\sigma) d \sigma .
\end{aligned}
$$

By using the Dirichlet formula, we have

$$
\begin{aligned}
\varepsilon^{\prime}(z)= & \int_{-1}^{z}\left(\frac{\partial f(z, \bar{\phi}(z))}{\partial f_{2}}+\int_{s}^{z}(z-\sigma)^{-\mu} \frac{\partial \widetilde{K}\left(z, \sigma, \bar{\varphi}(\sigma), y^{\prime}(\sigma)\right)}{\partial \widetilde{K}_{3}} d \sigma\right) \varepsilon^{\prime}(s) d s \\
& +\int_{-1}^{z}(z-\sigma)^{-\mu} \frac{\partial \widetilde{K}\left(z, \sigma, \bar{y}_{N}(\sigma), \bar{\psi}(\sigma)\right)}{\partial \widetilde{K}_{4}} \varepsilon^{\prime}(\sigma) d \sigma+\mathcal{Q}(z) .
\end{aligned}
$$

where

$$
\begin{aligned}
\mathcal{Q}(z)= & \int_{-1}^{z}(z-\sigma)^{-\mu} \frac{\partial \widetilde{K}\left(z, \sigma, \bar{\varphi}(\sigma), y^{\prime}(\sigma)\right)}{\partial \widetilde{K}_{3}}\left(Q_{2}(\sigma)+Q_{3}(\sigma)\right) d \sigma+Q_{1}(z) \\
& +\sum_{j=4}^{8} Q_{j}(z)+I_{N}^{\theta, v} L(z) .
\end{aligned}
$$

and

$$
\begin{aligned}
& \left|\frac{\partial f(z, \bar{\phi}(z))}{\partial f_{2}}+\int_{s}^{z}(z-\sigma)^{-\mu} \frac{\partial \widetilde{K}\left(z, \sigma, \bar{\varphi}(\sigma), y^{\prime}(\sigma)\right)}{\partial \widetilde{K}_{3}} d \sigma\right| \\
& \leq \max _{z \in[-1,1]}\left|\frac{\partial f(z, \bar{\phi}(z))}{\partial f_{2}}\right|+\max _{(z, \sigma) \in \mathcal{D}_{1}}\left|\frac{\partial \widetilde{K}\left(z, \sigma, \bar{\varphi}(\sigma), y^{\prime}(\sigma)\right)}{\partial \widetilde{K}_{3}}\right| \int_{s}^{z}(z-\sigma)^{-\mu} d \sigma \\
& \quad \leq \max _{z \in[-1,1]}\left|\frac{\partial f(z, \bar{\phi}(z))}{\partial f_{2}}\right|+\frac{2^{1-\mu}}{1-\mu} \max _{(z, \sigma) \in \mathcal{D}_{1}}\left|\frac{\partial \widetilde{K}\left(z, \sigma, \bar{\varphi}(\sigma), y^{\prime}(\sigma)\right)}{\partial \widetilde{K}_{3}}\right| \equiv M_{3} \\
& \left|\frac{\partial \widetilde{K}\left(z, \sigma, \bar{y}_{N}(\sigma), \bar{\psi}(\sigma)\right)}{\partial \widetilde{K}_{4}}\right| \leq \max _{(z, \sigma) \in \mathcal{D}_{1}}\left|\frac{\partial \widetilde{K}\left(z, \sigma, \bar{y}_{N}(\sigma), \bar{\psi}(\sigma)\right)}{\partial \widetilde{K}_{4}}\right| \equiv M_{4}
\end{aligned}
$$


Then, (68) is written as

$$
\left|\varepsilon^{\prime}(z)\right| \leq M_{3} \int_{-1}^{z}\left|\varepsilon^{\prime}(\sigma)\right| d \sigma+M_{4} \int_{-1}^{z}(z-\sigma)^{-\mu}\left|\varepsilon^{\prime}(\sigma)\right| d \sigma+|\mathcal{Q}(z)|
$$

By using (64), (69) and Lemma 3.6, we have

$$
\begin{aligned}
& \left\|\varepsilon^{\prime}\right\|_{\infty} \leq C\|\mathcal{Q}(z)\|_{\infty} \leq C \sum_{i=1}^{8}\left\|Q_{i}\right\|_{\infty}+\left\|I_{N}^{\theta, v} L(z)\right\|_{\infty}, \\
& \|\varepsilon\|_{\infty} \leq C\left\|\varepsilon^{\prime}\right\|_{\infty}+\left\|Q_{2}\right\|_{\infty}+\left\|Q_{3}\right\|_{\infty} \leq C \sum_{i=1}^{8}\left\|Q_{i}\right\|_{\infty}+\left\|I_{N}^{\theta, v} L(z)\right\|_{\infty} .
\end{aligned}
$$

Due to Lemma 3.2, we obtain

$$
\begin{aligned}
& \left\|Q_{1}\right\|_{\infty}=\left\|y^{\prime}-I_{N}^{\theta, v} y^{\prime}\right\|_{\infty} \leq\left\{\begin{array}{l}
C N^{\frac{1}{2}-m} \log N\left|y^{\prime}\right|_{H_{\omega^{c}}^{m ; N}}, \quad-1<\theta, v \leq-\frac{1}{2} \\
C N^{\gamma+1-m}\left|y^{\prime}\right|_{H_{\omega^{c}}^{m ; N}}, \quad \text { otherwise, } \gamma=\max \{\theta, v\},
\end{array}\right. \\
& \left\|Q_{2}\right\|_{\infty}=\left\|y-I_{N}^{\theta, v} y\right\|_{\infty} \leq\left\{\begin{array}{l}
C N^{\frac{1}{2}-m} \log N|y|_{H_{\omega^{c}}^{m ; N}}, \quad-1<\theta, v \leq-\frac{1}{2} \\
C N^{\gamma+1-m}|y|_{H_{\omega^{c}}^{m ; N}}, \quad \text { otherwise, } \gamma=\max \{\theta, v\}
\end{array}\right.
\end{aligned}
$$

By using Lemma 3.2 with $m=1$, we obtain

$$
\begin{aligned}
\left\|Q_{3}\right\|_{\infty} \leq & \left\{\begin{array}{l}
C N^{-\frac{1}{2}} \log N\left|\mathcal{H} \varepsilon^{\prime}\right|_{H_{\omega^{c}}^{1 ; N}}, \quad-1<\theta, v \leq-\frac{1}{2}, \\
C N^{\gamma}\left|\mathcal{H} \varepsilon^{\prime}\right|_{H_{\omega^{c}}^{1 ; N}}, \quad-\frac{1}{2}<\gamma<0,
\end{array}\right. \\
\leq & \left\{\begin{array}{l}
C N^{-\frac{1}{2}} \log N\left\|\varepsilon^{\prime}\right\|_{\infty}, \quad-1<\theta, v \leq-\frac{1}{2}, \\
C N^{\gamma}\left\|\varepsilon^{\prime}\right\|_{\infty}, \quad-\frac{1}{2}<\gamma<0 .
\end{array}\right.
\end{aligned}
$$

Then, we use Lemma 3.2 with $m=1$ and Lemma 3.8, yields

$$
\begin{aligned}
\left\|Q_{8}\right\|_{\infty} \leq & \left\{\begin{array}{l}
C N^{-\frac{1}{2}} \log N\left|\frac{\partial f(z, \bar{\phi}(z))}{\partial f_{2}} \int_{-1}^{z} \varepsilon^{\prime}(\sigma) d \sigma\right|_{H_{\omega^{c}}^{1 ; N}}, \quad-1<\theta, v \leq-\frac{1}{2}, \\
C N^{\gamma}\left|\frac{\partial f(z, \bar{\phi}(z))}{\partial f_{2}} \int_{-1}^{z} \varepsilon^{\prime}(\sigma) d \sigma\right|_{H_{\omega^{c}}^{1 ; N}}, \quad-\frac{1}{2}<\gamma<0,
\end{array}\right. \\
\leq & \left\{\begin{array}{l}
C N^{-\frac{1}{2}} \log N\left\|\varepsilon^{\prime}\right\|_{\infty}, \quad-1<\theta, v \leq-\frac{1}{2}, \\
C N^{\gamma}\left\|\varepsilon^{\prime}\right\|_{\infty}, \quad-\frac{1}{2}<\gamma<0,
\end{array}\right.
\end{aligned}
$$

Furthermore, applying the (22), Lemma 3.4 and Lemma 3.7, we have 


$$
\begin{aligned}
\left\|Q_{4}\right\|_{\infty} & =\left\|I_{N}^{\theta, v} \mathcal{K}_{3} \varepsilon-\mathcal{K}_{3} \varepsilon\right\|_{\infty}=\left\|\left(I_{N}^{\theta, v}-I\right)\left(\mathcal{K}_{3} \varepsilon-\mathfrak{L}_{N} \mathcal{K}_{3} \varepsilon\right)\right\|_{\infty} \\
& \leq\left(1+\left\|I_{N}^{\theta, v}\right\|_{\infty}\right)\left\|\mathcal{K}_{3} \varepsilon-\mathfrak{L}_{N} \mathcal{K}_{3} \varepsilon\right\|_{\infty} \leq\left(1+\left\|I_{N}^{\theta, v}\right\|_{\infty}\right) C N^{-\varrho}\left\|\mathcal{K}_{3} \varepsilon\right\|_{0, \varrho} \\
& \leq\left\{\begin{array}{l}
C N^{-\varrho} \log N\|\varepsilon\|_{\infty}, \quad-1<\theta, v \leq-\frac{1}{2}, \\
C N^{\gamma+\frac{1}{2}-\varrho}\|\varepsilon\|_{\infty}, \quad \text { otherwise, } \gamma=\max \{\theta, v\},
\end{array} \quad \max \left\{\frac{1}{2}+\gamma, 0\right\}<\varrho<1-\mu .\right.
\end{aligned}
$$

$$
\begin{aligned}
\left\|Q_{5}\right\|_{\infty} & =\left\|I_{N}^{\theta, v} \mathcal{K}_{4} \varepsilon^{\prime}-\mathcal{K}_{4} \varepsilon^{\prime}\right\|_{\infty}=\left\|\left(I_{N}^{\theta, v}-I\right)\left(\mathcal{K}_{4} \varepsilon^{\prime}-\mathfrak{L}_{N} \mathcal{K}_{4} \varepsilon^{\prime}\right)\right\|_{\infty} \\
& \leq\left(1+\left\|I_{N}^{\theta, v}\right\|_{\infty}\right)\left\|\mathcal{K}_{4} \varepsilon^{\prime}-\mathfrak{L}_{N} \mathcal{K}_{4} \varepsilon^{\prime}\right\|_{\infty} \leq\left(1+\left\|I_{N}^{\theta, v}\right\|_{\infty}\right) C N^{-\varrho}\left\|\mathcal{K}_{4} \varepsilon^{\prime}\right\|_{0, \varrho} \\
& \leq\left\{\begin{array}{l}
C N^{-\varrho} \log N\left\|\varepsilon^{\prime}\right\|_{\infty}, \quad-1<\theta, v \leq-\frac{1}{2}, \\
C N^{\gamma+\frac{1}{2}-\varrho}\left\|\varepsilon^{\prime}\right\|_{\infty}, \quad \text { otherwise, } \gamma=\max \{\theta, v\},
\end{array} \quad \max \left\{\frac{1}{2}+\gamma, 0\right\}<\varrho<1-\mu .\right.
\end{aligned}
$$

By employing Lemma 3.4 yields

$$
\begin{aligned}
\left\|Q_{6}\right\|_{\infty} \leq & \left\{\begin{array}{l}
C \log N\left\|\frac{\partial f(z, \bar{\phi}(z))}{\partial f_{2}} Q_{2}(z)\right\|_{\infty}, \quad-1<\theta, v \leq-\frac{1}{2}, \\
C N^{\gamma+\frac{1}{2}}\left\|\frac{\partial f(z, \bar{\phi}(z))}{\partial f_{2}} Q_{2}(z)\right\|_{\infty}, \quad \text { otherwise, } \gamma=\max \{\theta, v\},
\end{array}\right. \\
\leq & \left\{\begin{array}{l}
C N^{\frac{1}{2}-m} \log ^{2} N|y|_{H_{\omega^{c}}^{m ; N}}, \quad-1<\theta, v \leq-\frac{1}{2}, \\
C N^{2 \gamma+\frac{3}{2}-m}|y|_{H_{\omega^{c}}^{m ; N}}, \quad \text { otherwise, } \gamma=\max \{\theta, v\} .
\end{array}\right. \\
\left\|Q_{7}\right\|_{\infty} \leq & \left\{\begin{array}{l}
C \log N\left\|\frac{\partial f(z, \bar{\phi}(z))}{\partial f_{2}} Q_{3}(z)\right\|_{\infty}, \quad-1<\theta, v \leq-\frac{1}{2}, \\
C N^{\gamma+\frac{1}{2}}\left\|\frac{\partial f(z, \bar{\phi}(z))}{\partial f_{2}} Q_{3}(z)\right\|_{\infty}, \quad-\frac{1}{2}<\gamma<-\frac{1}{4},
\end{array}\right. \\
\leq & \left\{\begin{array}{l}
C N^{-\frac{1}{2}} \log ^{2} N\left\|\varepsilon^{\prime}\right\|_{\infty}, \quad-1<\theta, v \leq-\frac{1}{2}, \\
C N^{2 \gamma+\frac{1}{2}}\left\|\varepsilon^{\prime}\right\|_{\infty},-\frac{1}{2}<\gamma<-\frac{1}{4} .
\end{array}\right.
\end{aligned}
$$

Next, we apply the definition of the semi-norms $|\cdot|_{W_{\omega^{-}}^{m, 0}}^{m ; N}$ and Lemma 3.5, we obtain

$$
\begin{aligned}
& |L(z)|=\left|\left(\mathcal{K}\left(\bar{y}_{N}, \bar{y}_{N}^{\prime}\right)\right)(z)-\left(\mathcal{K}_{N}\left(\bar{y}_{N}, \bar{y}_{N}^{\prime}\right)\right)(z)\right| \\
& \leq C N^{-m}\left|\widetilde{K}\left(z, \sigma(z, \cdot), \bar{y}_{N}(\sigma(z, \cdot)), \bar{y}_{N}^{\prime}(\sigma(z, \cdot))\right)\right|_{H_{\omega^{-\mu, 0}}^{m ; N}} \\
& =C N^{-m}\left(\sum_{p=\min (m, N+1)}^{m}\left\|\frac{\partial^{p} \widetilde{K}\left(z, \sigma(z, \cdot), \bar{y}_{N}(\sigma(z, \cdot)), \bar{y}_{N}^{\prime}(\sigma(z, \cdot))\right)}{\partial \zeta^{p}}\right\|_{\omega^{-\mu, 0}}^{2}\right)^{\frac{1}{2}}
\end{aligned}
$$


and

$$
\begin{aligned}
& \left|\frac{\partial^{p} \widetilde{K}\left(z, \sigma(z, \zeta), \bar{y}_{N}(\sigma(z, \zeta)), \bar{y}_{N}^{\prime}(\sigma(z, \zeta))\right)}{\partial \zeta^{p}}\right| \\
& =\left|\left(\frac{\partial \sigma}{\partial \zeta}\right)^{p} \cdot \frac{\partial^{p} \widetilde{K}\left(z, \sigma, \bar{y}_{N}(\sigma), \bar{y}_{N}^{\prime}(\sigma)\right)}{\partial \sigma^{p}}\right| \leq\left|\frac{\partial^{p} \widetilde{K}\left(z, \sigma, \bar{y}_{N}(\sigma), \bar{y}_{N}^{\prime}(\sigma)\right)}{\partial \sigma^{p}}\right| \\
& \leq\left|\frac{\partial^{p} \widetilde{K}\left(z, \sigma, y(\sigma), y^{\prime}(\sigma)\right)}{\partial \sigma^{p}}\right|+\left|\frac{\partial^{p} \widetilde{K}\left(z, \sigma, \bar{y}_{N}(\sigma), \bar{y}_{N}^{\prime}(\sigma)\right)}{\partial \sigma^{p}}-\frac{\partial^{p} \widetilde{K}\left(z, \sigma, y(\sigma), y^{\prime}(\sigma)\right)}{\partial \sigma^{p}}\right|
\end{aligned}
$$

Using the Lipschitz continuous condition, we have

$$
\begin{aligned}
& \left|\widetilde{K}\left(z, \sigma, \bar{y}_{N}(\sigma), \bar{y}_{N}^{\prime}(\sigma)\right)-\widetilde{K}\left(z, \sigma, y(\sigma), y^{\prime}(\sigma)\right)\right|_{\substack{H_{\omega^{-\mu, 0}}^{m ; N}\\
}}=\left(\sum_{p=\min (m, N+1)}^{m}\left\|\frac{\partial^{p} \widetilde{K}\left(z, \sigma, \bar{y}_{N}(\sigma), \bar{y}_{N}^{\prime}(\sigma)\right)}{\partial \sigma^{p}}-\frac{\partial^{p} \widetilde{K}\left(z, \sigma, y(\sigma), y^{\prime}(\sigma)\right)}{\partial \sigma^{p}}\right\|_{\omega^{-\mu, 0}}^{2}\right)^{\frac{1}{2}} \\
& \quad \leq \sum_{p=\min (m, N+1)}^{m}\left(\sum_{q=0}^{1} L_{p q}\left\|\bar{y}_{N}^{(q)}-y^{(q)}\right\|_{\omega^{-\mu, 0}}\right) \\
& \leq C\left(\left\|\bar{y}_{N}-y\right\|_{\omega^{-\mu, 0}}+\left\|\bar{y}_{N}^{\prime}-y^{\prime}\right\|_{\omega^{-\mu, 0}}\right) .
\end{aligned}
$$

Hence, (79) can be rewritten as

$$
\begin{aligned}
& |L(z)| \leq C N^{-m}\left|\widetilde{K}\left(z, \sigma, y(\sigma), y^{\prime}(\sigma)\right)\right|_{H_{\omega^{-\mu, 0}}^{m ; N}} \\
& +C N^{-m}\left|\widetilde{K}\left(z, \sigma, \bar{y}_{N}(\sigma), \bar{y}_{N}^{\prime}(\sigma)\right)-\widetilde{K}\left(z, \sigma, y(\sigma), y^{\prime}(\sigma)\right)\right|_{\substack{H^{m}-\mu, 0 \\
m, N}} \\
& \leq C N^{-m}\left(\left|\tilde{K}\left(z, \sigma, y(\sigma), y^{\prime}(\sigma)\right)\right|_{H_{\omega^{-\mu, 0}}^{m ; N}}+\|\varepsilon\|_{\omega^{-\mu, 0}}+\left\|\varepsilon^{\prime}\right\|_{\omega^{-\mu, 0}}\right)
\end{aligned}
$$

By using Lemma 3.4, we get

$$
\begin{aligned}
\left\|I_{N}^{\theta, v} L(z)\right\|_{\infty} & \leq\left\{\begin{array}{l}
C \log N\|L(z)\|_{\infty}, \quad-1<\theta, v \leq-\frac{1}{2}, \\
C N^{\gamma+\frac{1}{2}}\|L(z)\|_{\infty}, \quad \text { otherwise, } \gamma=\max \{\theta, v\},
\end{array}\right. \\
& \leq\left\{\begin{array}{l}
C N^{-m} \log N\left(\mathcal{K}^{*}+\|\varepsilon\|_{\omega^{-\mu, 0}}+\left\|\varepsilon^{\prime}\right\|_{\omega^{-\mu, 0}}\right), \quad-1<\theta, v \leq-\frac{1}{2}, \\
C N^{\gamma+\frac{1}{2}-m}\left(\mathcal{K}^{*}+\|\varepsilon\|_{\omega^{-\mu, 0}}+\left\|\varepsilon^{\prime}\right\|_{\omega^{-\mu, 0}}\right), \quad \text { otherwise, } \gamma=\max \{\theta, v\},
\end{array}\right. \\
& \leq\left\{\begin{array}{l}
C N^{-m} \log N\left(\mathcal{K}^{*}+\|\varepsilon\|_{\infty}+\left\|\varepsilon^{\prime}\right\|_{\infty}\right), \quad-1<\theta, v \leq-\frac{1}{2}, \\
C N^{\gamma+\frac{1}{2}-m}\left(\mathcal{K}^{*}+\|\varepsilon\|_{\infty}+\left\|\varepsilon^{\prime}\right\|_{\infty}\right), \quad \text { otherwise, } \gamma=\max \{\theta, v\} .
\end{array}\right.
\end{aligned}
$$

where $\mathcal{K}^{*}=\max _{z \in[-1,1]}\left|\widetilde{K}\left(z, \sigma, y(\sigma), y^{\prime}(\sigma)\right)\right|_{H_{\omega^{-\mu, 0}}^{m ; N}}$. 
On the other hand, by using (64), (69), Lemma 3.6 and Lemma 3.8, we have

$$
\begin{aligned}
& \left\|\varepsilon^{\prime}\right\|_{\omega^{\theta, v}} \leq C \sum_{i=1}^{8}\left\|Q_{i}\right\|_{\omega^{\theta, v}}+\left\|I_{N}^{\theta, v} L(z)\right\|_{\omega^{\theta, v}}, \\
& \|\varepsilon\|_{\omega^{\theta, v}} \leq C\left\|\varepsilon^{\prime}\right\|_{\omega^{\theta, v}}+\left\|Q_{2}\right\|_{\omega^{\theta, v}}+\left\|Q_{3}\right\|_{\omega^{\theta, v}} \leq C \sum_{i=1}^{8}\left\|Q_{i}\right\|_{\omega^{\theta, v}}+\left\|I_{N}^{\theta, v} L(z)\right\|_{\omega^{\theta, v}} .
\end{aligned}
$$

Due to Lemma 3.2, we have

$$
\begin{aligned}
& \left\|Q_{1}\right\|_{\omega^{\theta, v}}=\left\|y^{\prime}-I_{N}^{\theta, v} y^{\prime}\right\|_{\omega^{\theta, v}} \leq C N^{-m}\left|y^{\prime}\right|_{H_{\omega^{\theta, v}}^{m ; N}}, \\
& \left\|Q_{2}\right\|_{\omega^{\theta, v}}=\left\|y-I_{N}^{\theta, v} y\right\|_{\omega^{\theta, v}} \leq C N^{-m}|y|_{\substack{H_{\omega^{\theta, v}}^{m ; N} \\
\text { m, }}},
\end{aligned}
$$

By applying Lemma 3.2 with $m=1$ yields

$$
\left\|Q_{3}\right\|_{\omega^{\theta, v}}=\left\|I_{N}^{\theta, \nu} \mathcal{H} \varepsilon^{\prime}-\mathcal{H} \varepsilon^{\prime}\right\|_{\omega^{\theta, v}} \leq C N^{-1}\left|\mathcal{H} \varepsilon^{\prime}\right|_{H_{\omega^{\theta, v}}^{1 ; N}} \leq C N^{-1}\left\|\varepsilon^{\prime}\right\|_{\omega^{\theta, \nu}} .
$$

Moreover, in the virtue of Lemma 3.2 with $m=1$ and Lemma 3.8, we obtain

$$
\left\|Q_{8}\right\|_{\omega^{\theta, \nu}} \leq C N^{-1}\left|\frac{\partial f(z, \bar{\phi}(z))}{\partial f_{2}} \int_{-1}^{z} \varepsilon^{\prime}(\sigma) d \sigma\right|_{H_{\omega^{\theta, v}}^{1 ; N}} \leq C N^{-1}\left\|\varepsilon^{\prime}\right\|_{\omega^{\theta, v}} .
$$

Next, by applying (22), Lemma 3.3 and Lemma 3.7, we have

$$
\begin{aligned}
\left\|Q_{4}\right\|_{\omega^{\theta, v}} & =\left\|I_{N}^{\theta, v} \mathcal{K}_{3} \varepsilon-\mathcal{K}_{3} \varepsilon\right\|_{\omega^{\theta, v}}=\left\|\left(I_{N}^{\theta, v}-I\right)\left(\mathcal{K}_{3} \varepsilon-\mathfrak{L}_{N} \mathcal{K}_{3} \varepsilon\right)\right\|_{\omega^{\theta, v}} \\
& \leq\left\|I_{N}^{\theta, v}\left(\mathcal{K}_{3} \varepsilon-\mathfrak{L}_{N} \mathcal{K}_{3} \varepsilon\right)\right\|_{\omega^{\theta, v}}+\left\|\mathcal{K}_{3} \varepsilon-\mathfrak{L}_{N} \mathcal{K}_{3} \varepsilon\right\|_{\omega^{\theta, v}} \\
& \leq C\left\|\mathcal{K}_{3} \varepsilon-\mathfrak{L}_{N} \mathcal{K}_{3} \varepsilon\right\|_{\infty} \leq C N^{-\varrho}\left\|\mathcal{K}_{3} \varepsilon\right\|_{0, \varrho} \leq C N^{-\varrho}\|\varepsilon\|_{\infty} . \\
\left\|Q_{5}\right\|_{\omega^{\theta, v}} & =\left\|I_{N}^{\theta, v} \mathcal{K}_{4} \varepsilon^{\prime}-\mathcal{K}_{4} \varepsilon^{\prime}\right\|_{\omega^{\theta, v}}=\left\|\left(I_{N}^{\theta, v}-I\right)\left(\mathcal{K}_{4} \varepsilon^{\prime}-\mathfrak{L}_{N} \mathcal{K}_{4} \varepsilon^{\prime}\right)\right\|_{\omega^{\theta, v}} \\
& \leq\left\|I_{N}^{\theta, v}\left(\mathcal{K}_{4} \varepsilon^{\prime}-\mathfrak{L}_{N} \mathcal{K}_{4} \varepsilon^{\prime}\right)\right\|_{\omega^{\theta, v}}+\left\|\mathcal{K}_{4} \varepsilon^{\prime}-\mathfrak{L}_{N} \mathcal{K}_{4} \varepsilon^{\prime}\right\|_{\omega^{\theta, v}} \\
& \leq C\left\|\mathcal{K}_{4} \varepsilon^{\prime}-\mathfrak{L}_{N} \mathcal{K}_{4} \varepsilon^{\prime}\right\|_{\infty} \leq C N^{-\varrho}\left\|\mathcal{K}_{4} \varepsilon^{\prime}\right\|_{0, \varrho} \leq C N^{-\varrho}\left\|\varepsilon^{\prime}\right\|_{\infty} .
\end{aligned}
$$

Then, we use Lemma 3.3 yields

$$
\begin{aligned}
& \left\|Q_{6}\right\|_{\omega^{\theta, v}} \leq C\left\|\frac{\partial f(z, \bar{\phi}(z))}{\partial f_{2}} Q_{2}(z)\right\|_{\infty} \\
& \leq\left\{\begin{array}{l}
C N^{\frac{1}{2}-m} \log N|y|_{H_{\omega^{c}}^{m ; N}}, \quad-1<\theta, v \leq-\frac{1}{2}, \\
C N^{\gamma+1-m}|y|_{H_{\omega^{c}}^{m ; N}}, \quad \text { otherwise, } \gamma=\max \{\theta, \nu\},
\end{array}\right. \\
& \left\|Q_{7}\right\|_{\omega^{\theta, v}} \leq C\left\|\frac{\partial f(z, \bar{\phi}(z))}{\partial f_{2}} Q_{3}(z)\right\|_{\infty} \\
& \leq\left\{\begin{array}{l}
C N^{-\frac{1}{2}} \log N\left\|\varepsilon^{\prime}\right\|_{\infty}, \quad-1<\theta, v \leq-\frac{1}{2}, \\
C N^{\gamma}\left\|\varepsilon^{\prime}\right\|_{\infty}, \quad-\frac{1}{2}<\gamma<0
\end{array}\right.
\end{aligned}
$$


By using the Lemma 3.3 and (82), we have

$$
\begin{aligned}
\left\|I_{N}^{\theta, v} L(z)\right\|_{\omega^{\theta, v}} & \leq C\|L(z)\|_{\infty} \leq C \max _{z \in[-1,1]}|L(z)| \\
& \leq C N^{-m}\left(\mathcal{K}^{*}+\|\varepsilon\|_{\omega^{-\mu, 0}}+\left\|\varepsilon^{\prime}\right\|_{\omega^{-\mu, 0}}\right) \\
& \leq C N^{-m}\left(\mathcal{K}^{*}+\|\varepsilon\|_{\infty}+\left\|\varepsilon^{\prime}\right\|_{\infty}\right)
\end{aligned}
$$

When the provided $N$ is large enough, we obtain the desired estimate (58), (59).

\section{Numerical examples}

For this section, we have implemented several test problems to confirm the theoretical results. In addition, we will compare the results of our proposed method with other numerical methods. MAPLE software is used to implement the programs corresponding to our proposed technique and fsolve command is implemented to solve final SNAEs. Due to the complexity of numerical examples, we employ the Jacobi pseudo-spectral Galerkin method (JPSGM) to solve the following test problems. In all examples, $L^{\infty}$ and $L^{2}$-norms are defined as follows:

$$
\|e\|_{L^{\infty}}=\max _{x \in[-1,1]}|e(x)|, \quad\|e\|_{L^{2}}=\left(\int_{-1}^{1} e^{2}(x) d x\right)^{\frac{1}{2}} .
$$

Example 5.1 we study the nonlinear weakly singular Volterra integro-differential equations (NWSVIDEs) as follows:

$$
\begin{aligned}
& u^{\prime}(t)=g(t, u(t))+\int_{0}^{t}(t-\eta)^{-\frac{1}{3}} K\left(t, \eta, u(\eta), u^{\prime}(\eta)\right) d \eta, \quad t \in[0,8], \\
& u(0)=e^{-1}
\end{aligned}
$$

where

$$
\begin{aligned}
g(t, u(t)) & =\frac{1}{3} t^{-\frac{2}{3}} u(t)-\frac{9 \Gamma\left(\frac{2}{3}\right)^{3} \sqrt{3}+4 \pi^{2} \sqrt{3}+9 \pi}{54 \pi}\left(t^{\frac{1}{3}}-1\right)^{2}, \\
K\left(t, \eta, u(\eta), u^{\prime}(\eta)\right) & =\frac{1}{9} t^{-\frac{2}{3}} \eta^{-\frac{2}{3}}\left(t^{\frac{2}{3}}+t^{\frac{1}{3}} \eta^{\frac{1}{3}}+\eta^{\frac{2}{3}}\right)\left(t^{\frac{1}{3}}-1\right)^{2}\left(e^{-2\left(\eta^{\frac{1}{3}}-1\right)} u^{2}(\eta)\right) .
\end{aligned}
$$

and the exact solution $u(t)=e^{\sqrt[3]{t}-1}$.

Observing the solution of this equations, we can find that its first derivative $u^{\prime}(t)=\frac{e^{\sqrt[3]{t}-1}}{3 t^{\frac{2}{3}}}$ is unbounded at $t=0$, that is, $u^{\prime}(t)$ is discontinuous at $t=0$. Therefore, in order to use our method to solve the equation, first use a smooth transformation to deal with the original equation, namely: set $t=s^{3}, \eta=\xi^{3}$, we have

$$
\begin{aligned}
\hat{u}^{\prime}(s) & =\hat{g}(s, \hat{u}(s))+\int_{0}^{s}(s-\xi)^{-\frac{1}{3}} \hat{K}\left(s, \xi, \hat{u}(\xi), \hat{u}^{\prime}(\xi)\right) d \xi, \quad s \in[0,2], \\
\hat{u}(0) & =e^{-1}
\end{aligned}
$$

then

$$
\hat{g}(s, \hat{u}(s))=\hat{u}(s)-\frac{9 \Gamma\left(\frac{2}{3}\right)^{3} \sqrt{3}+4 \pi^{2} \sqrt{3}+9 \pi}{18 \pi} s^{2}(s-1)^{2},
$$


Table 1 The corresponding errors in $L^{\infty}$ and $L_{\omega}^{2}$ for Example 5.1

\begin{tabular}{lcccc}
\hline$N$ & $\left\|y-y_{N}\right\|_{L_{\omega}^{2}}$ & $\left\|y-y_{N}\right\|_{L_{\infty}}$ & $\left\|y^{\prime}-y_{N}^{\prime}\right\|_{L_{\omega}^{2}}$ & $\left\|y^{\prime}-y_{N}^{\prime}\right\|_{L_{\infty}}$ \\
\hline 2 & $3.9084 e-02$ & $7.3361 e-02$ & $3.9333 e-02$ & $8.9789 e-02$ \\
4 & $9.1986 e-04$ & $2.9624 e-03$ & $2.8553 e-03$ & $8.4144 e-03$ \\
6 & $3.1383 e-06$ & $9.0718 e-06$ & $5.8937 e-06$ & $1.6455 e-05$ \\
8 & $1.0258 e-08$ & $2.5892 e-08$ & $1.3923 e-08$ & $3.1560 e-08$ \\
10 & $2.3099 e-11$ & $5.6929 e-11$ & $2.8531 e-11$ & $5.9831 e-11$ \\
12 & $9.7006 e-13$ & $2.1294 e-12$ & $2.3607 e-12$ & $6.8498 e-12$ \\
\hline
\end{tabular}

$$
\hat{K}\left(s, \xi, \hat{u}(\xi), \hat{u}^{\prime}(\xi)\right)=\left(s^{2}+s \xi+\xi^{2}\right)^{\frac{2}{3}}(s-1)^{2}\left(e^{-2(\xi-1)} \hat{u}^{2}(\xi)\right) .
$$

and $\hat{u}(s)=e^{s-1}$ is smooth.

Furthermore, by applying the appropriate transformation to change the solution domain to the standard interval $[-1,1]$. Set $s=1+z, \xi=1+\sigma$, yields

$$
\begin{aligned}
y^{\prime}(z) & =f(z, y(z))+\int_{-1}^{z}(z-\sigma)^{-\frac{1}{3}} \widetilde{K}\left(z, \sigma, y(\sigma), y^{\prime}(\sigma)\right) d \sigma, \\
y(-1) & =e^{-1}, \quad z \in[-1,1] .
\end{aligned}
$$

where

$$
\begin{gathered}
f(z, y(z))=y(z)-\frac{9 \Gamma\left(\frac{2}{3}\right)^{3} \sqrt{3}+4 \pi^{2} \sqrt{3}+9 \pi}{18 \pi}(1+z)^{2} z^{2}, \\
\widetilde{K}\left(z, \sigma, y(\sigma), y^{\prime}(\sigma)\right)=\left((1+z)^{2}+(1+z)(1+\sigma)+(1+\sigma)^{2}\right)^{\frac{2}{3}} z^{2}\left(e^{-2 \sigma} y^{2}(\sigma)\right) .
\end{gathered}
$$

and $y(z)=e^{z}$.

Next, we use JPSGM to solve this problem. Table 1 shows the numerical error for $N=$ $2,4, \ldots, 12$ in both $L^{\infty}$ and $L_{\omega}^{2}$ norms. And we also demonstrate the corresponding numerical errors in Fig. 1, it can be clearly observed that the errors decay exponentially. Moreover, the left of Fig. 2 displays the approximate solution of JPSGM for $N=12$ and the exact solution of $u(t)$, and also the right of Fig. 2 demonstrates the approximate derivative and the exact derivative of $u^{\prime}(t)$. Figure 2 illustrates that the solution obtained by the smooth transformation can be inversely transformed back to the solution of the original equation. This also verifies the effectiveness of the methods.

Example 5.2 As another test problem, we discuss NWSVIDEs as follows:

$$
\begin{aligned}
& u^{\prime}(t)=\frac{7}{5} t^{\frac{2}{5}}-\frac{\Gamma\left(\frac{2}{5}\right) \Gamma\left(\frac{19}{5}\right)}{\Gamma\left(\frac{21}{5}\right)} t^{\frac{16}{5}}+\int_{0}^{t}(t-\eta)^{-\frac{3}{5}} u^{2}(\eta) d \eta, \quad t \in[0,1], \\
& u(0)=0 .
\end{aligned}
$$

with $u(t)=t^{\frac{7}{5}}$.

In Wang et al. (2017), the authors presented the Legendre-Jacobi spectral collocation method of hp-version for this problem and derived the error bounds of the hp-version collocation method under the $H^{1}$-norm. As the solution $u(t)$ exists a weakly singularity at 

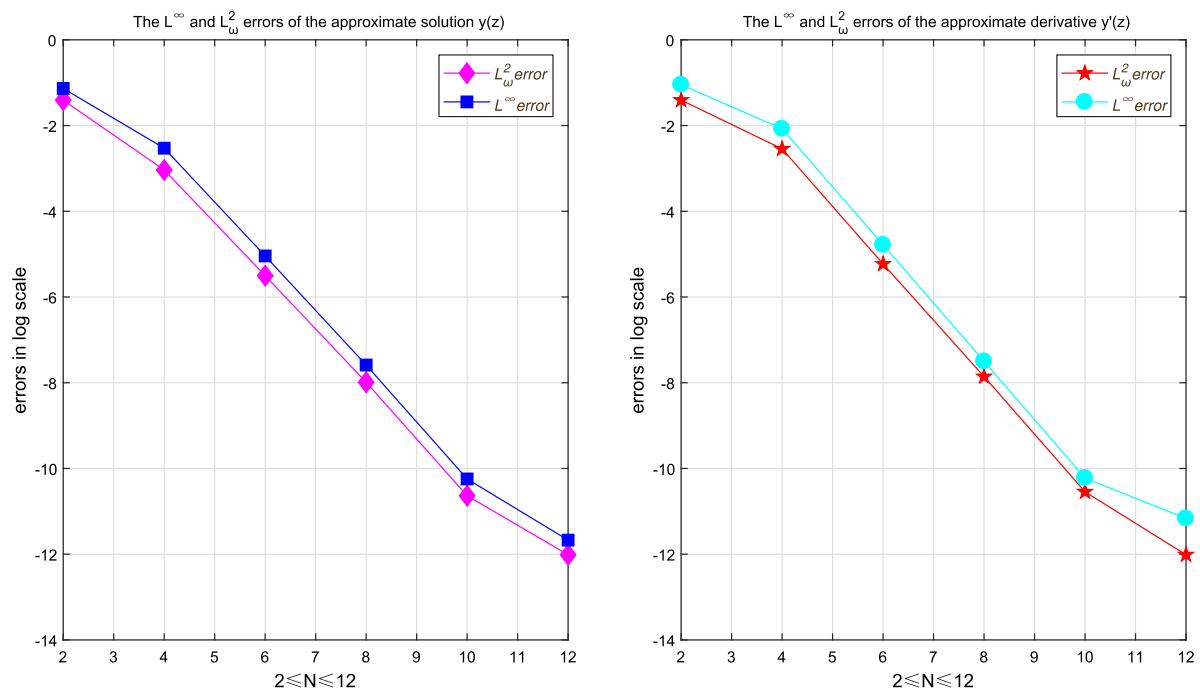

Fig. 1 The $L^{\infty}$ and $L_{\omega}^{2}$ errors of the approximate solution of $y(z)$ (left) and the approximate derivative of $y^{\prime}(z)$ (right) for Example 5.1
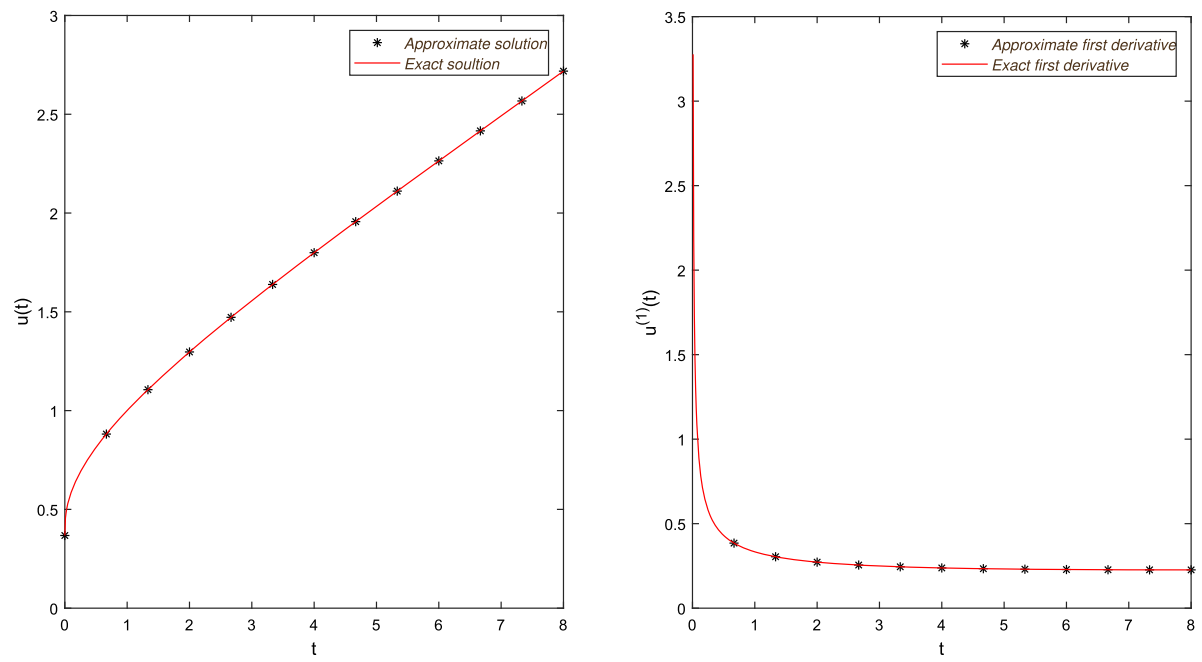

Fig. 2 The exact solution $u(t)$ and the approximate solution (left). And also the exact derivative $u^{\prime}(t)$ and the approximate derivative (right) for Example 5.1

$t=0$. Thus, we apply a variable transformation to this equation such that the solution of the transformed equation is sufficiently smooth. And it can be solved by the JPSGM.

Therefore, set $t=\left(\frac{1+z}{2}\right)^{5}, \eta=\left(\frac{1+\sigma}{2}\right)^{5}$, we obtain

$y^{\prime}(z)=\frac{7}{2^{7}}(1+z)^{6}-\frac{5}{2^{21}}(1+z)^{20} \frac{\Gamma\left(\frac{2}{5}\right) \Gamma\left(\frac{19}{5}\right)}{\Gamma\left(\frac{21}{5}\right)}+\int_{-1}^{z}(z-\sigma)^{-\frac{3}{5}} \widetilde{K}\left(z, \sigma, y(\sigma), y^{\prime}(\sigma)\right) d \sigma$,

$y(-1)=0, \quad z \in[-1,1]$. 
Table 2 The corresponding errors in $L^{\infty}$ and $L_{\omega}^{2}$ for Example 5.2

\begin{tabular}{lllll}
\hline$N$ & $\left\|y-y_{N}\right\|_{L_{\omega}^{2}}$ & $\left\|y-y_{N}\right\|_{L_{\infty}}$ & $\left\|y^{\prime}-y_{N}^{\prime}\right\|_{L_{\omega}^{2}}$ & $\left\|y^{\prime}-y_{N}^{\prime}\right\|_{L_{\infty}}$ \\
\hline 4 & $1.2738 e-02$ & $1.9724 e-02$ & $2.3812 e-02$ & $3.0367 e-02$ \\
6 & $1.2307 e-04$ & $2.1464 e-04$ & $5.3997 e-05$ & $1.8207 e-04$ \\
8 & $2.2804 e-12$ & $1.1361 e-11$ & $2.8978 e-11$ & $1.4222 e-10$ \\
10 & $5.1029 e-15$ & $2.5634 e-14$ & $6.5001 e-14$ & $3.2873 e-13$ \\
12 & $6.6462 e-16$ & $2.3804 e-15$ & $2.7643 e-15$ & $1.4082 e-14$ \\
14 & $2.0185 e-15$ & $7.2491 e-15$ & $8.4837 e-15$ & $4.3303 e-14$ \\
\hline
\end{tabular}

where

$$
\begin{aligned}
\widetilde{K}\left(z, \sigma, y(\sigma), y^{\prime}(\sigma)\right)= & \frac{25}{2^{7}}(1+z)^{4}(1+\sigma)^{4} y^{2}(\sigma) . \\
& \left((1+z)^{4}+(1+z)^{3}(1+\sigma)+(1+z)^{2}(1+\sigma)^{2}\right. \\
& \left.\left.+(1+z)(1+\sigma)^{3}+(1+\sigma)^{4}\right)\right)^{-\frac{3}{5}}
\end{aligned}
$$

and $y(z)=\left(\frac{1+z}{2}\right)^{7}$.

Furthermore, the following Table and Figures show the results solved by the proposed method. This example is implemented on Maple and takes digits $=20$. In Table 2, we list the numerical errors in both $L^{\infty}$ and $L_{\omega}^{2}$ norms. And we also draw the relevant numerical errors with various values $N$ in Fig. 3. As the value of $N$ increases, the error decreases. It should be noticed that the exponential convergence is implemented. Moreover, the left of Fig. 4 shows the approximate solution of JPSGM for $N=14$ and the exact solution $u(t)$, and also the right of Fig. 4 shows the approximate derivative and the exact derivative $u^{\prime}(t)$. Figure 4 shows that the numerical solution of the inverse transformation is very close to the true solution of the original equation. This means that the solution of the equation after the transformation can be obtained through smooth transformation first, and then back to the solution of the original equation through the inverse transformation.

Example 5.3 For the last test problem, we research the system of NWSVIDEs as follows:

$$
\begin{aligned}
& u_{1}^{\prime}(t)=\frac{1}{30 \sqrt{t}}\left(15-30 t-40 t^{4}-32 t^{5}\right)+u_{1}(t)+t^{2} \int_{0}^{t}(t-\eta)^{-\frac{1}{2}} \eta \cdot u_{2}^{2}(\eta) d \eta, \\
& u_{2}^{\prime}(t)=-0.675 t^{\frac{11}{3}}-\frac{1+2 t}{2 \sqrt{1+t}}+u_{2}(t)+t \int_{0}^{t}(t-\eta)^{-\frac{1}{3}} \eta \cdot u_{1}^{2}(\eta) d \eta, \\
& u_{1}(0)=0, \quad u_{2}(0)=1,
\end{aligned}
$$

with $u_{1}(t)=\sqrt{t}, u_{2}(t)=\sqrt{1+t}$.

In Sahu and Ray (2016), the authors use Bernoulli wavelet method (BWM) to solve the system of NWSVIDEs. The wavelet method is used to simplify the integro-differential equations into a nonlinear algebraic equations, and then the Newton method is applied to numerically solve the nonlinear algebraic equations. Example 5.3 is a numerical example in this article. By observing the exact solutions $u_{1}(t)$ and $u_{2}(t)$, we can find that $u_{1}(t)$ is not smooth in the given domain while $u_{2}(t)$ is smooth. Therefore, according to the expression of $u_{1}(t)$, we use the variable transformation to this equation, such that the solution of the transformed equation is sufficiently smooth. Set $t=\left(\frac{1+z}{2}\right)^{2}, \eta=\left(\frac{1+\sigma}{2}\right)^{2}$, we have 

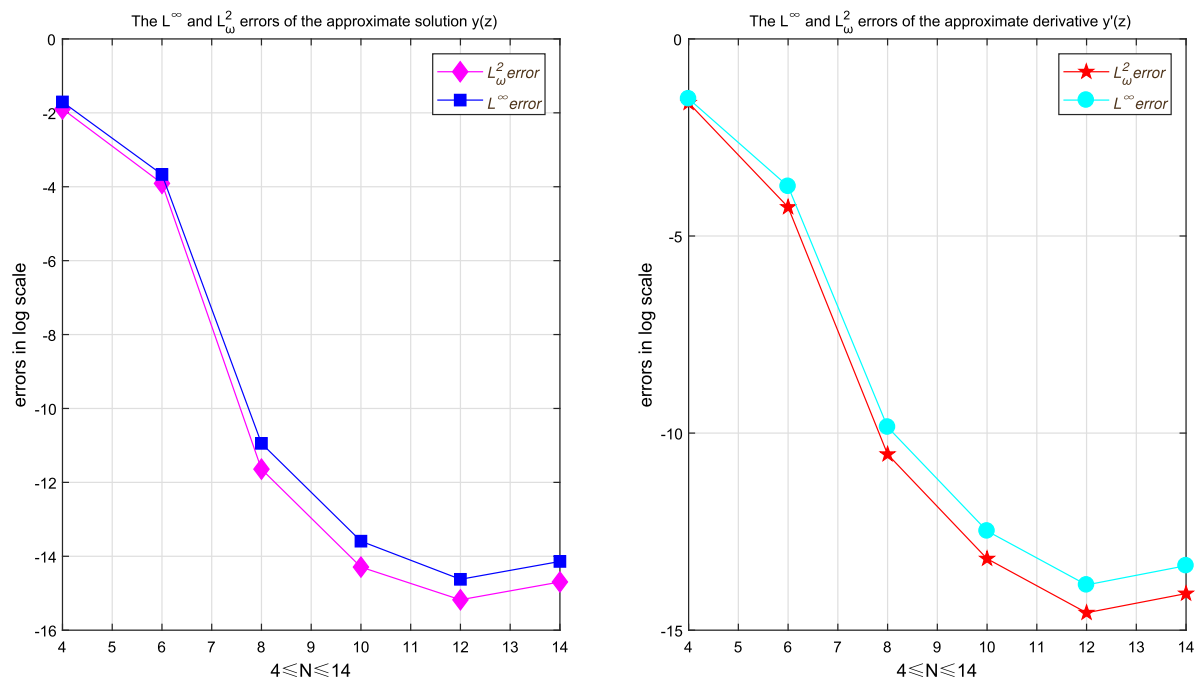

Fig. 3 The $L^{\infty}$ and $L_{\omega}^{2}$ errors of numerical solution of $y(z)$ (left) and the approximate derivative of $y^{\prime}(z)$ (right) for Example 5.2
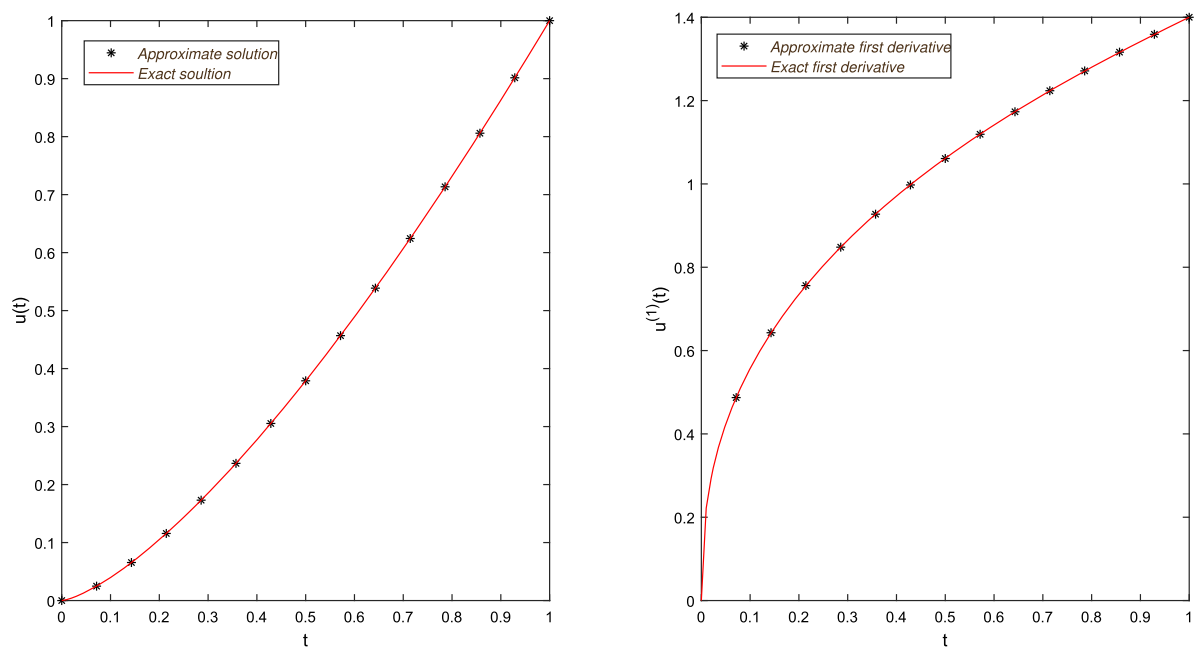

Fig. 4 Comparison between $u(t)$ and the approximate solution (left). And also the comparison between $u^{\prime}(t)$ and the approximate derivative (right) for Example 5.2

$$
\begin{aligned}
& y_{1}^{\prime}(z)=f_{1}\left(z, y_{1}(z)\right)+\frac{(1+z)^{5}}{2^{7}} \int_{-1}^{z}(z-\sigma)^{-\frac{1}{2}}(2+z+\sigma)^{-\frac{1}{2}}(1+\sigma)^{3} y_{2}^{2}(\sigma) d \sigma \\
& y_{2}^{\prime}(z)=f_{2}\left(z, y_{2}(z)\right)+\frac{(1+z)^{3}}{2^{\frac{16}{3}}} \int_{-1}^{z}(z-\sigma)^{-\frac{1}{3}}(2+z+\sigma)^{-\frac{1}{3}}(1+\sigma)^{3} y_{1}^{2}(\sigma) d \sigma, \quad z \in[-1,1], \\
& y_{1}(-1)=0, \quad y_{2}(-1)=1
\end{aligned}
$$


Table 3 The numerical solution results of $u_{1}(t)$ and absolute errors for Example 5.3

\begin{tabular}{llllll}
\hline$t$ & Exact solution $u_{1}(t)$ & BWMSahu and Ray (2016) & Absolute errors & JPSGM & Absolute errors \\
\hline 0.1 & 0.316228 & 0.314381 & $1.8463 e-3$ & 0.3162278 & $4.296 e-9$ \\
0.2 & 0.447214 & 0.449175 & $1.9613 e-3$ & 0.4472136 & $8.386 e-10$ \\
0.3 & 0.547723 & 0.549819 & $2.0965 e-3$ & 0.5477226 & $6.139 e-9$ \\
0.4 & 0.632456 & 0.633161 & $7.050 e-4$ & 0.6324555 & $3.908 e-9$ \\
0.5 & 0.707107 & 0.709594 & $2.4876 e-3$ & 0.7071068 & $5.117 e-9$ \\
0.6 & 0.774597 & 0.777067 & $2.4705 e-3$ & 0.7745967 & $1.243 e-8$ \\
0.7 & 0.836660 & 0.839433 & $2.7728 e-3$ & 0.836660 & $1.068 e-8$ \\
0.8 & 0.894427 & 0.897514 & $3.0869 e-3$ & 0.8944272 & $1.535 e-9$ \\
0.9 & 0.948683 & 0.952134 & $3.4507 e-3$ & 0.9486833 & $1.642 e-8$ \\
1.0 & 1.000000 & 1.00412 & $4.1154 e-3$ & 0.9999999 & $1.487 e-8$ \\
\hline
\end{tabular}

Table 4 The numerical solution results of $u_{2}(t)$ and absolute errors for Example 5.3

\begin{tabular}{llllll}
\hline$t$ & Exact solution $u_{2}(t)$ & BWMSahu and Ray (2016) & Absolute errors & JPSGM & Absolute errors \\
\hline 0.1 & 1.04881 & 1.0488 & $4.09 e-6$ & 1.048809 & $3.91 e-7$ \\
0.2 & 1.09545 & 1.09545 & $1.82 e-6$ & 1.095445 & $3.42 e-7$ \\
0.3 & 1.14018 & 1.14018 & $5.75 e-6$ & 1.140175 & $2.39 e-7$ \\
0.4 & 1.18322 & 1.18323 & $1.04 e-5$ & 1.183216 & $3.07 e-7$ \\
0.5 & 1.22474 & 1.22479 & $4.45 e-5$ & 1.224745 & $3.83 e-7$ \\
0.6 & 1.26491 & 1.26502 & $1.10 e-4$ & 1.264911 & $5.92 e-8$ \\
0.7 & 1.30384 & 1.30407 & $2.26 e-4$ & 1.303840 & $4.29 e-7$ \\
0.8 & 1.34164 & 1.34206 & $4.22 e-4$ & 1.341641 & $1.90 e-7$ \\
0.9 & 1.37840 & 1.37915 & $7.41 e-4$ & 1.378405 & $3.88 e-7$ \\
1.0 & 1.41421 & 1.41545 & $1.24 e-3$ & 1.414213 & $4.30 e-7$
\end{tabular}

where

$$
\begin{aligned}
f_{1}\left(z, y_{1}(z)\right)= & \frac{1}{2}-\left(\frac{1+z}{2}\right)^{2}-\frac{4}{3} \cdot\left(\frac{1+z}{2}\right)^{8}-\frac{16}{15} \cdot\left(\frac{1+z}{2}\right)^{10}+\frac{1+z}{2} y_{1}(z), \\
f_{2}\left(z, y_{2}(z)\right)= & -0.675 \cdot\left(\frac{1+z}{2}\right)^{\frac{25}{3}}-\left(\frac{1+z}{4}+\left(\frac{1+z}{2}\right)^{3}\right) \cdot\left(1+\left(\frac{1+z}{2}\right)^{2}\right)^{-\frac{1}{2}} \\
& +\frac{1+z}{2} y_{2}(z)
\end{aligned}
$$

with $y_{1}(z)=\frac{1+z}{2}, y_{2}(z)=\left(1+\left(\frac{1+z}{2}\right)^{2}\right)^{\frac{1}{2}}$.

Next, we use JPSGM to solve the transformed equation and show the relevant numerical results in Tables and Figures. Example 5.3 has been solved by BWM with $k=2$ and $M=4$ in Sahu and Ray (2016), that is, it applied $2^{k-1} M$ Bernoulli wavelets to approximate the unknown functions $u_{1}(t)$ and $u_{2}(t)$, respectively. In order to compare with this method, we approximate the unknown function with the same number of Jacobi basis functions. Thus, we take $N=7$ and use the JPSGM to solve Example 5.3. And then, Tables 3, 4 present the 

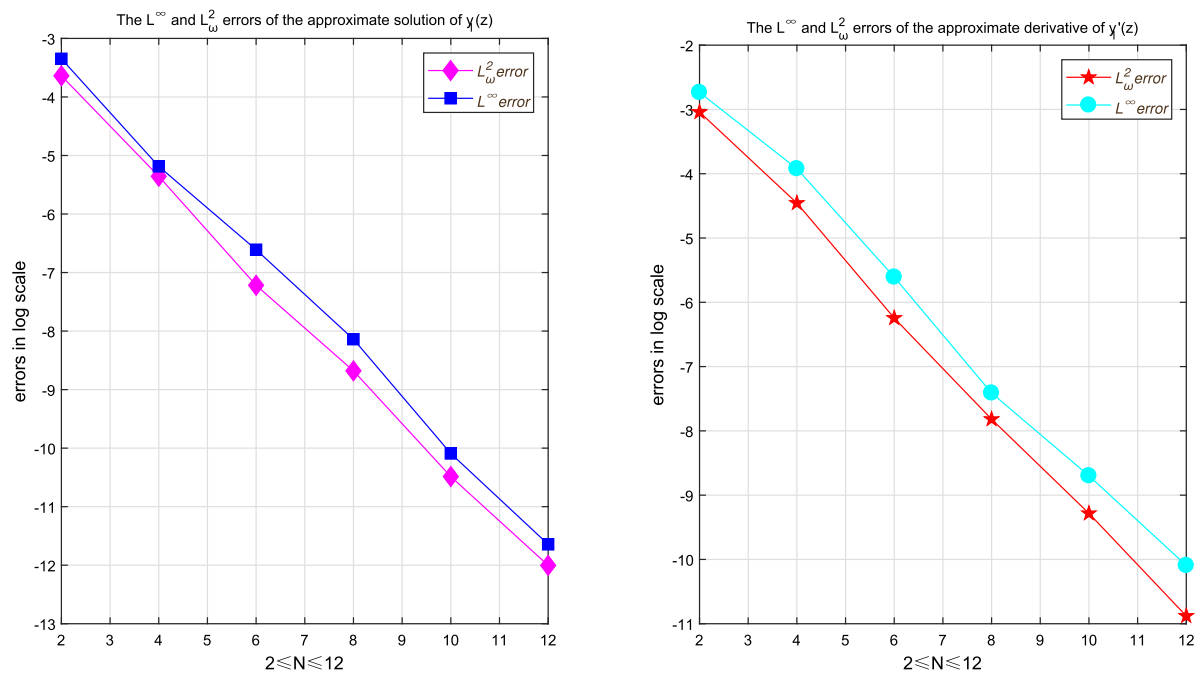

Fig. 5 The $L^{\infty}$ and $L_{\omega}^{2}$ errors of approximate solution of $y_{1}(z)$ (left) and the approximate derivative of $y_{1}^{\prime}(z)$ (right) for Example 5.3
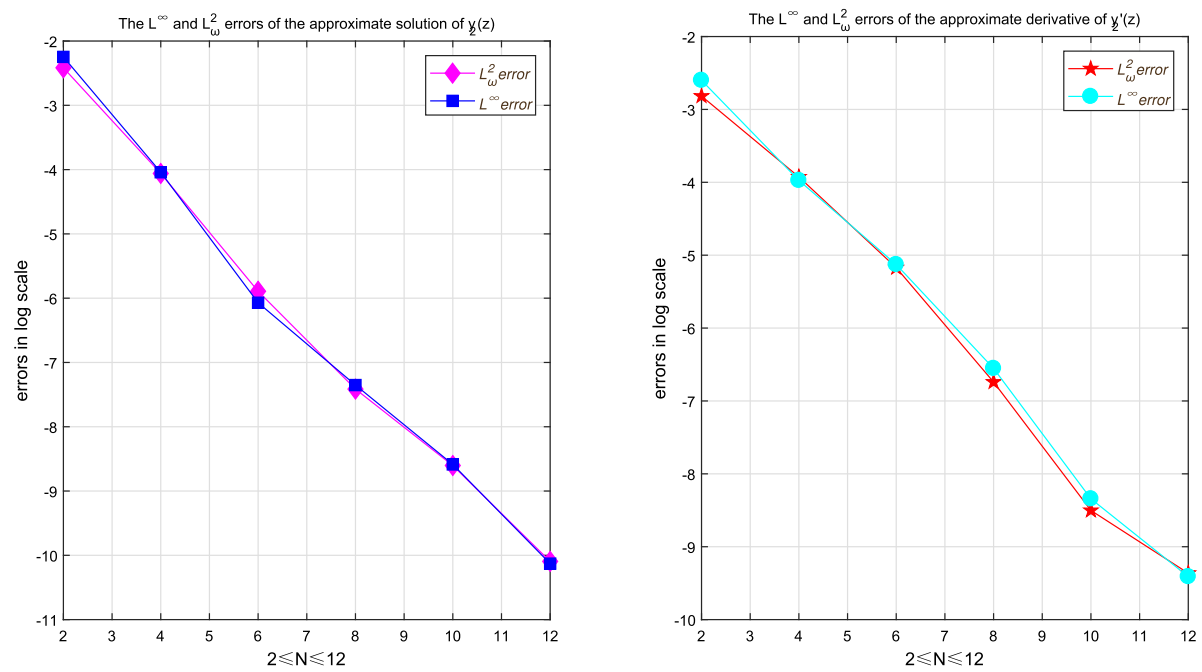

Fig. 6 The $L^{\infty}$ and $L_{\omega}^{2}$ errors of approximate solution of $y_{2}(z)$ (left) and the approximate derivative of $y_{2}^{\prime}(z)$ (right) for Example 5.3

comparison of the numerical results of our method and BWM in this case. It is clear that our method is better than BWM to approximate the exact solutions in this case. In addition, the $L^{\infty}$ and $L_{\omega}^{2}$ errors of the approximate solutions and also the approximate derivatives have been shown for different values of $N$ in the Figs. 5, 6. It can be seen intuitively from the Figures that the errors decay exponentially. Furthermore, Figs. 7, 8 show the comparison between the numerical solutions of the inverse transformation and the exact solutions, the numerical derivatives of the inverse transformation and the exact derivatives. It confirms that 

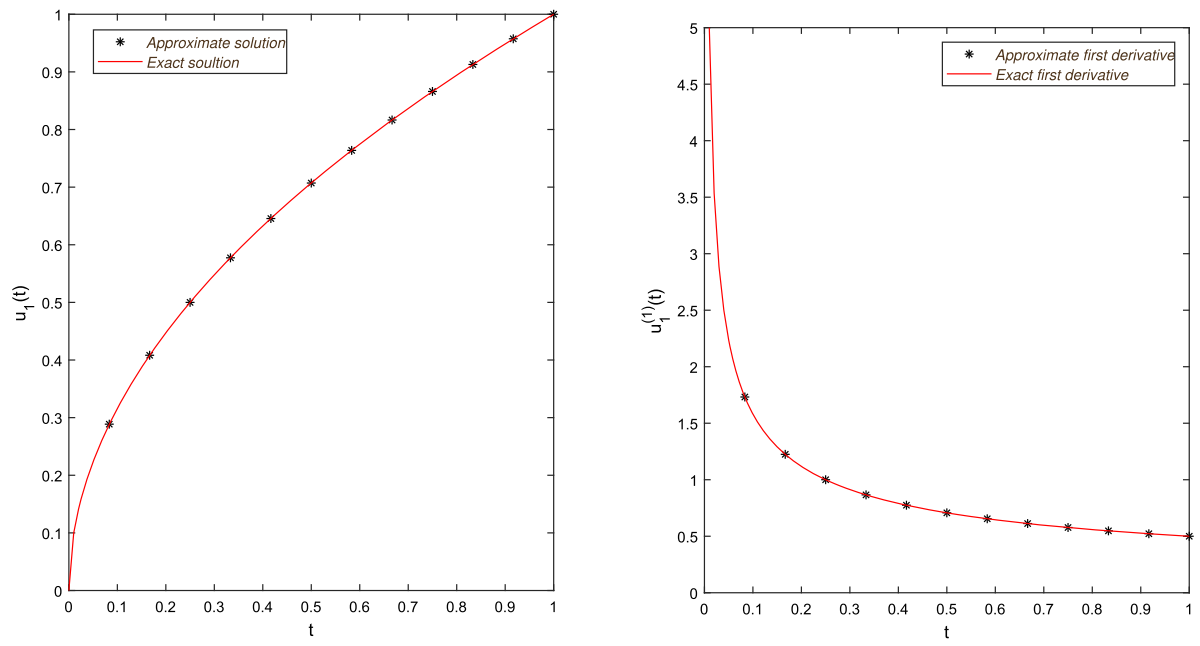

Fig. 7 Comparison between $u_{1}(t)$ and the approximate solution (left). And also the comparison between $u_{1}^{\prime}(t)$ and the approximate derivative (right) for Example 5.3
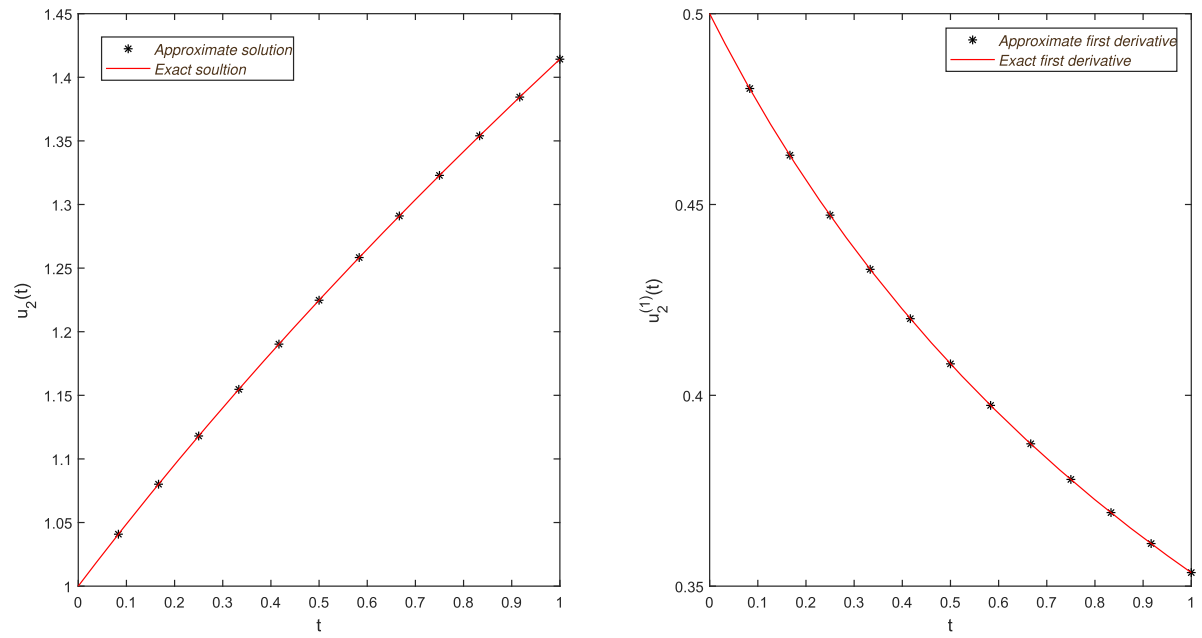

Fig. 8 Comparison between $u_{2}(t)$ and the approximate solution (left). And also the comparison between $u_{2}^{\prime}(t)$ and the approximate derivative (right) for Example 5.3

the numerical solutions of the equation after transformation can be transformed back to the numerical solutions of the original equation by inverse transformation.

\section{Conclusions}

In this research article, both of the spectral and pseudo-spectral Jacobi Galerkin approaches are investigated in detail for computing the numerical solutions of a general form of nonlinear WSVIDEs of the first order. Before implementing these numerical methods, some appropriate changes of variables are considered to make the solutions of the equations to be smooth. By 
these changes of variables, new equations may have better regularity behaviour. In the case of Jacobi pseudo-spectral Galerkin technique, Jacobi Gauss quadrature rules are used in both the operator approximations and also inner products approximations. Exponential rate of convergence corresponding to the suggested approaches in both $L^{\infty}$ and weighted $L^{2}$ norms are also discussed in details. Superior numerical results of the suggested approaches with respect to some new global numerical methods motivate us to apply these methods in a multi-step version instead of single-step one. This subject will be our future choice in the next research works.

Acknowledgements This work was supported by the National Natural Science Foundation of China Project (12071402, 11931003), the National Key Research and Development Program of China (2020YFA0713503), the Project of Scientific Research Fund of the Hunan Provincial Science and Technology Department (2020JJ2027, 2020ZYT003, 2018WK4006).

\section{Declarations}

Conflict of interest The authors state that they do not have any conflict of interest in their submitted manuscript.

\section{References}

Baratella P, Orsi AP (2004) A new approach to the numerical solution of weakly singular Volterra integral equations. J Comput Appl Math 163(2):401-418

Brunner H (2004) Collocation methods for Volterra integral and related functional differential equations, vol 15. Cambridge University Press, Cambridge

Canuto CG, Hussaini MY, Quarteroni A, Zang T (2006) Spectral methods fundamentals in single domains. Springer, Berlin

Chen Y, Tang T (2009) Spectral methods for weakly singular Volterra integral equations with smooth solutions. J Comput Appl Math 233(4):938-950

Chen Y, Tang T (2010) Convergence analysis of the Jacobi spectral-collocation methods for Volterra integral equation with a weakly singular kernel. Math Comput 79(269):147-167

Douglas J, Dupont T, Wahlbin L (1974) The stability in $L^{q}$ of the $L^{2}$-projection into finite element function spaces. Numerische Mathematik 23(3):193-197

Gao W, Veeresha P, Prakasha DG, Baskonus HM, Yel G (2020) New approach for the model describing the deathly disease in pregnant women using Mittag-Leffler function. Chaos Solitons Fractals 134:109696

Gao W, Veeresha P, Baskonus HM, Prakasha DG, Kumar P (2020) A new study of unreported cases of 2019-nCOV epidemic outbreaks. Chaos Solitons Fractals 138:109929

Kunfer A, Persson LE (2003) Weighted inequalities of Hardy type. World Scientific, New York

Lü T, Huang Y (2006) Extrapolation method for solving weakly singular nonlinear Volterra integral equations of the second kind. J Math Anal Appl 324:225-237

Mastroianni G, Occorsio D (2001) Optimal systems of nodes of for Lagrange interpolation on bounded interals. a survey. J Comput Appl Math 134(1-2):325-341

Ragozin D (1970) Polynomial approximation on compact manifolds and homogeneous space. Trans Am Math Soc 150:41-53

Ragozin D (1971) Constructive polynomial approximation on spheres and projective spaces. Trans Am Math Soc 162:157-170

Sahu PK, Ray SS (2016) A New Bernoulli Wavelet Method for Numerical Solutions of Nonlinear Weakly Singular Volterra Integro-Differential Equations. Int J Comput Methods 1750022

Shen J, Tang T, Wang LL (2011) Spectral methods. Algorithms, analysis and applications. Springer, New York

Shi X, Wei Y, Huang F (2019) Spectral collocation methods for nonlinear weakly singular Volterra integrodifferential equations. Numer Methods Partial Differ Equ 35:576-596

Sohrabi S, Ranjbar H, Saei M (2017) Convergence analysis of the Jacobi-collocation method for nonlinear weakly singular Volterra integral equations. App Math Comput 299:141-152

Tang T, Xiang XU, Cheng J (2008) On spectral methods for Volterra integral equations and the convergence analysis. J Comput Math 26(6):825-837 
Tao X, Xie Z, Zhou X (2011) Spectral Petrov-Galerkin Methods for the Second Kind Volterra Type IntegroDifferential Equations. Numer Math Theor Meth Appl 4(002):216-236

Wang CL, Wang ZQ, Jia HL (2017) An hp-version Spectral Collocation Method for Nonlinear Volterra Integro-differential Equation with Weakly Singular Kernels. J Sci Comput 72(2):1-32

Wei Y, Chen Y (2012) Convergence analysis of the spectral methods for weakly singular Volterra integrodifferential equations with smooth solutions. Adv Appl Math Mech 4(01):1-20

Willett D (1965) A linear generalization of Gronwall's inequality. Proc Am Math Soc 16(4):774-778

Yang Y (2015) Jacobi spectral Galerkin methods for fractional integro-differential equations. Calcolo 52:519_ 542

Yang Y, Tang Z (2021) Mapped Spectral collocation methods for Volterra integral equations with noncompact kernels. Appl Numer Math 160:166-177

Yang X, Yang Y, Chen Y, Liu J (2018) Jacobi spectral collocation method based on Lagrange interpolation polynomials for solving nonlinear fractional integro-differential equations. Adv Appl Math Mech 10(6):1440-1458

Yang Y, Tang Z, Huang Y (2019) Numerical solutions for Fredholm integral equations of the second kind with weakly singular kernel using spectral collocation method. Appl Math Comput 349:314-324

Yang Y, Tohidi E, Ma X, Kang S (2019) Rigorous convergence analysis of Jacobi spectral Galerkin methods for Volterra integral equations with noncompact kernels. J Comput Appl Math 366:112403

Yel G, Baskonus HM, Gao W (2020) New dark-bright soliton in the shallow water wave model. AIMS Math 5(4):4027-4044

Zhang X, Li J (2014) Jacobi pseudo-spectral Galerkin method for second kind Volterra integro-differential equations with a weakly singular kernel. J Vibroeng 16(8):3807-3826

Zhang R, Zhu B, Xie H (2013) Spectral methods for weakly singular Volterra integral equations with pantograph delays. Front Math China 8(002):281-299

Publisher's Note Springer Nature remains neutral with regard to jurisdictional claims in published maps and institutional affiliations. 